\title{
Fuzzy aura matrices for texture classification
}

\author{
Kamal Hammouche ${ }^{\mathrm{a}}$, Olivier Losson ${ }^{\mathrm{b}, *}$, Ludovic Macaire ${ }^{\mathrm{b}}$ \\ a Laboratoire Vision Artificielle et Automatique des Systèmes, Université Mouloud Mammeri, Tizi-Ouzou, Algérie \\ ${ }^{b}$ Laboratoire CRIStAL (UMR CNRS 9189), Université de Lille - Sciences et Technologies, \\ Cité Scientifique - Bâtiment P2, 59655 Villeneuve d'Ascq Cedex, France
}

\begin{abstract}
The aura concept has been developed from the set theory and is an efficient tool to characterize texture images. It is based on the notion of "aura set" and on the associated "aura measure" that involve the neighborhood of each image pixel. In this paper, we propose to extend this concept to the framework of fuzzy sets in order to take the imprecise nature of images into account. We define the notions of fuzzy aura sets and of aura measures to compute fuzzy aura matrices as texture descriptors. Fuzzy aura measures assume no restrictions about the neighborhood shape, size, and spatial invariance. Extensive tests of texture classification on Outex benchmark datasets show that fuzzy aura matrices computed with spatially-variant neighborhoods often outperform other powerful texture descriptors on both gray-level and color images.

Keywords: Fuzzy aura set, Fuzzy aura matrix, Texture classification, Spatially-variant neighborhood
\end{abstract}

\section{Introduction}

The classification of texture images is a fundamental problem in computer vision [1] and many texture features that characterize the relationships between gray levels of neighboring sites have been proposed for texture classification [2]. Among them, those derived from Gray-Level Co-occurrence Matrices (GLCMs) are very popular [3] because they provide good texture classification results [4]. A GLCM well characterizes textures because it gathers all the spatial co-occurrences of the pairs of sites associated to all the pairs of gray levels of an image.

Gray-Level Aura Matrices (GLAMs) proposed by Elfadel and Picard [5] rely on a set-theoretic concept called the "aura set" which describes the relative presence of a set of sites with a given gray level in the neighborhood of another set of sites with another gray level. An aura set can be characterized by a number called "aura measure" that expresses the amount of mixing between the two neighboring site sets. A GLAM gathers all the aura measures of the pairs of site sets associated to all the pairs of gray levels present in an image.

With Elfadel and Picard's aura measure, GLAMs can be viewed as a generalization of GLCMs and their computation schemes are very similar. Formally, a co-occurrence is defined according to

*Corresponding author. Tel.: +33-3 204341 69, fax: +33-320434245.

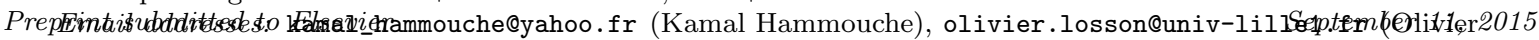
Losson), ludovic.macaire@univ-lille1.fr (Ludovic Macaire) 
the direction and distance between two sites, whereas an aura measure uses a neighborhood with any shape and size. A co-occurrence corresponds to a site-based viewpoint, whereas an aura measure is based on sets of sites and provides an interpretation of gray-level co-occurrences as set boundaries. GLAMs are therefore used for a few years as new tools for texture representation and synthesis $[6,7]$, and image retrieval [8, 9], classification [10-15] and segmentation [16]. However, the original aura measure produces GLAMs that do not strictly represent the aura sets of an image. In this paper, we first propose a new cardinal-based measure and experimentally show that it better characterizes the aura sets for texture classification purposes.

Digital images carry more or less fuzziness because of the imprecision inherent to the discretization of both the spatial domain (sampling) and the levels (quantization). Hence, boundaries separating the various image regions are not precisely defined and the site levels are imprecise measures of the reflectance of the surfaces observed by the camera. Furthermore, the hypothesis that texture images are mainly represented by spatial repetitions of a pattern may not be valid any longer. Image processing techniques based on fuzzy concepts have then been proposed in order to take all these imprecisions into account [17-19] and fuzzy GLCMs have been designed to characterize texture images [20-23]. Another contribution of this paper is a generalization of aura measures to the fuzzy framework. We consider both Elfadel and Picard's aura measure and our cardinal-based one to characterize fuzzy aura sets, and show that fuzzy GLAMs are more relevant descriptors of texture images than GLCMs.

All the works about the aura set cited before follow Elfadel and Picard's seminal proposal and consider spatially-invariant neighborhoods. The underlying assumption is that the neighborhood of each site is the translate of a generic neighborhood, as for structuring elements used by mathematical morphology. The last contribution of this paper is to show that the aura set theory can drop this restriction by defining neighborhoods that are specific to each site. In the context of fuzzy sets, we illustrate that adaptive neighborhoods are useful for image analysis, with texture classification as an example. Such interest is investigated by Verdú-Monedero et al. [24] and by Landström and Thurley [25] with a formulation using mathematical morphology. We show that the aura definitions provide an elegant formalism as well to deal with spatially-variant neighborhoods and that the derived measures efficiently hold such adaptability.

The rest of the paper is organized as follows. The second section recalls some definitions and properties of the aura concept in the context of crisp sets, and explains how to apply it to describe textures by GLAMs. In the third section, we extend these definitions to fuzzy sets and propose the fuzzy GLAMs to overcome the limitations of crisp aura sets for texture analysis. The fourth section highlights how spatially-variant neighborhoods can be useful to represent images by fuzzy GLAMs in different cases of image degradation. By reporting experimental results, the fifth section shows the benefits of the proposed approach for texture classification. 


\section{Crisp aura}

In this section, we recall some definitions in relation to the aura concept applied to a gray-level image, namely the aura set, aura measure, and gray-level aura matrix. More details about the properties and demonstrations of the notions given below can be found in [5]. Measures derived from the aura concept are used to characterize the spatial distribution of the gray levels. We show the limitations of these measures to recognize gray-level texture images when acquisition conditions vary.

\subsection{Aura sets of a gray-level image}

To define an image, let us consider a finite lattice $\mathcal{S}$ fitted with a neighborhood structure $\mathfrak{N}=$ $\left\{\mathcal{N}_{\mathbf{s}}, \mathbf{s} \in \mathcal{S}\right\}$, where $\mathcal{N}_{\mathbf{s}}$ is a neighborhood of the site $\mathbf{s}$. Let us now consider a gray-level image $I$ quantized with $q$ levels and defined on $\mathcal{S}$, such that each site $\mathbf{s} \in \mathcal{S}$ is characterized by its gray level $I(\mathbf{s})$. We call gray-level set the set $S_{g} \subseteq \mathcal{S}$ of sites with a given gray level $g, 0 \leq g \leq q-1$, namely $S_{g}=\{\mathbf{s} \in \mathcal{S}, I(\mathbf{s})=g\}$. Then, $\left\{S_{g}, 0 \leq g \leq q-1\right\}$ is a partition of $\mathcal{S}$ such that $\bigcup_{g=0}^{q-1} S_{g}=\mathcal{S}$ and $S_{g} \cap S_{g^{\prime}}=\emptyset$ for $g \neq g^{\prime}$.

Definition 1. Let $S_{g}$ and $S_{g^{\prime}}$ be two gray-level sets. The aura set $\mathcal{O}_{S_{g^{\prime}}}\left(S_{g}\right)$ of $S_{g}$ with respect to $S_{g^{\prime}}$ for the neighborhood structure $\mathfrak{N}$ is defined by $[5]^{1}$ :

$$
\mathcal{O}_{S_{g^{\prime}}}\left(S_{g}\right)=\bigcup_{\mathbf{s} \in S_{g}}\left(\mathcal{N}_{\mathbf{s}} \cap S_{g^{\prime}}\right)
$$

It is the subset of $S_{g^{\prime}}$ composed of the sites that are present in the neighborhood of those of $S_{g}$. It provides an interpretation of the presence of $S_{g^{\prime}}$ in the neighborhood of $S_{g}$.

Figure 1(a) shows a gray-level image defined on a $5 \times 5$ lattice $\mathcal{S}$ and two gray-level sets $S_{2}$ and $S_{3}$. For the neighborhood $\mathcal{N}_{\mathbf{s}}$ defined identically at any site $\mathbf{s}$ as shown in figure $1(\mathrm{~b})$, the aura set $\mathcal{O}_{S_{3}}\left(S_{2}\right)$ of $S_{2}$ with respect to $S_{3}$ is composed of the sites marked as boxes in figure 1(c). Note that it differs from the aura set $\mathcal{O}_{S_{2}}\left(S_{3}\right)$ of $S_{3}$ with respect to $S_{2}$, composed of the sites marked as diamonds in figure $1(\mathrm{~d})$.

When the neighborhood $\mathcal{N}_{\mathbf{s}}$ is defined identically at any site $\mathbf{s}$, it can be viewed as a translation at each site of a generic neighborhood denoted as $\mathcal{N}$ and called the structuring element. The aura set of a gray-level set $S_{g}$ with respect to $S_{g^{\prime}}$ can then be defined using the basic morphological dilation operator $\oplus$ as the intersection between $S_{g^{\prime}}$ and $S_{g}$ dilated by $\mathcal{N}[5]$ :

$$
\mathcal{O}_{S_{g^{\prime}}}\left(S_{g}\right)=\left(S_{g} \oplus \mathcal{N}\right) \cap S_{g^{\prime}}
$$

\footnotetext{
${ }^{1}$ Like Elfadel and Picard, we omit the notational dependency of the aura set on $\mathfrak{N}$ because this neighborhood structure is given once and for all.
} 


\begin{tabular}{|l|l|l|l|l|l|}
\hline 3 & 0 & 3 & 0 & 1 \\
\hline \hline 0 & 3 & 2 & 3 & 0 \\
\hline 2 & 3 & 2 & 2 & 3 \\
\hline 0 & 3 & 2 & 3 & 0 \\
\hline 2 & 0 & 3 & 0 & 1 \\
\hline
\end{tabular}

(a) Gray-level sets

$S_{2}$ (diamonds) and $S_{3}$ (boxes)

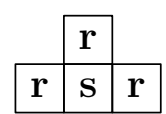

(b) Neighborhood $\mathcal{N}_{\mathbf{s}}$

(s: site of interest, $\mathbf{r}$ : neighbors)

\begin{tabular}{|l|l|l|l|l|}
\hline 3 & 0 & 3 & 0 & 1 \\
\hline 0 & 3 & 2 & 3 & 0 \\
\hline 2 & 3 & 2 & 2 & 3 \\
\hline 0 & 3 & 2 & 3 & 0 \\
\hline 2 & 0 & 3 & 0 & 1 \\
\hline
\end{tabular}

(c) Aura set

$\mathcal{O}_{S_{3}}\left(S_{2}\right)$ (boxes)

\begin{tabular}{|l|l|l|l|l|}
\hline 3 & 0 & 3 & 0 & 1 \\
\hline 0 & 3 & 2 & 3 & 0 \\
\hline 2 & 3 & 2 & 2 & 3 \\
\hline 0 & 3 & 2 & 3 & 0 \\
\hline 2 & 0 & 3 & 0 & 1 \\
\hline
\end{tabular}

(d) Aura set

$\mathcal{O}_{S_{2}}\left(S_{3}\right)$ (diamonds)

\begin{tabular}{|l|l|l|l|l|}
\hline 3 & 0 & 3 & 0 & 1 \\
\hline 0 & $3 \rightarrow$ & -2 & -3 & 0 \\
\hline $2-3 \rightarrow 2$ & 2 & -3 \\
\hline 0 & 3 & -2 & -3 & 0 \\
\hline 2 & 0 & 3 & 0 & 1 \\
\hline
\end{tabular}

(e) Local-count aura measure $m_{l}(2,3)$

(arrows)

Figure 1: Examples of two aura sets on a simple gray-level image.

\subsection{Aura measures and gray-level aura matrices}

Elfadel and Picard [5] define the aura measure as a function on the lattice $\mathcal{S}$ that characterizes an aura set. The gray-level aura matrix is a representation of the aura measures for all the possible pairs of gray-level sets in the lattice. This compact representation can be used as a descriptor of gray-level texture images. In the following, we present two aura measures that characterize the size of an aura set, and their associated aura matrices.

\subsubsection{Local-count aura measure and aura matrix}

The aura measure proposed by Elfadel and Picard is defined as the number of times that a site of $S_{g^{\prime}}$ is in the neighborhood of a site of $S_{g}$. To get the measure value for two given gray-level sets $S_{g}$ and $S_{g^{\prime}}$ and a given neighborhood structure $\mathfrak{N}$, one needs to examine the neighborhood $\mathcal{N}_{\mathbf{s}}$ of each site $\mathbf{s}$ of $S_{g}$ and to count the sites of $S_{g^{\prime}}$. We therefore refer to Elfadel and Picard's measure as the local-count aura measure and denote it as $m_{l}\left(g, g^{\prime}\right)$.

Definition 2. The local-count aura measure of a gray-level set $S_{g}$ with respect to a set $S_{g^{\prime}}$ according to the neighborhood structure $\mathfrak{N}=\left\{\mathcal{N}_{\mathbf{s}}, \mathbf{s} \in \mathcal{S}\right\}$ is defined as:

$$
m_{l}\left(g, g^{\prime}\right)=\sum_{\mathbf{s} \in S_{g}}\left|\mathcal{N}_{\mathbf{s}} \cap S_{g^{\prime}}\right|
$$

where $|\cdot|$ is the cardinal operator. The corresponding local-count GLAM $\left(\operatorname{GLAM}_{l}\right)$, denoted as $M_{l}$, is the $q \times q$ integer matrix defined by $M_{l}=\left[m_{l}\left(g, g^{\prime}\right)\right], 0 \leq g, g^{\prime} \leq q-1$.

As noted by Elfadel and Picard [5], the local-count aura matrix is closely related to the gray-level co-occurrence matrix (GLCM) defined as the $q \times q$ matrix $C=\left[c^{\mathbf{t}}\left(g, g^{\prime}\right)\right]$ whose element $\left(g, g^{\prime}\right)$ is the number of pairs of sites with gray levels $g$ and $g^{\prime}$ that are related by a translation vector $\mathbf{t}[3]$ :

$$
c^{\mathbf{t}}\left(g, g^{\prime}\right)=\left|(\mathbf{s}, \mathbf{s}+\mathbf{t}) \in \mathcal{S}^{2},(I(\mathbf{s})=g) \wedge\left(I(\mathbf{s}+\mathbf{t})=g^{\prime}\right)\right| .
$$


Indeed, when the neighborhood $\mathcal{N}_{\mathbf{s}} \equiv \mathcal{N}_{\mathbf{s}}^{\mathbf{t}}=\{\mathbf{s}+\mathbf{t}\}$ specifically contains only one neighbor, a GLAM ${ }_{l}$ is identical to a GLCM because $m_{l}\left(g, g^{\prime}\right)$ can be interpreted as the number of co-occurrences of sites $\mathbf{s}$ with gray level $g$ and neighboring sites $\mathbf{s}+\mathbf{t}$ with gray level $g^{\prime}[5]$. When $\mathcal{N}_{\mathbf{s}}$ is more generally composed of $n$ neighbors, it can be decomposed as $\mathcal{N}_{\mathbf{s}}=\bigcup_{i=1}^{n} \mathcal{N}_{\mathbf{s}}^{\mathbf{t}_{i}}$ into $n$ disjoint basic neighborhoods $\mathcal{N}_{\mathbf{s}}^{\mathbf{t}_{i}}$, each containing only one neighbor given by its translation $\mathbf{t}_{i}$ from $\mathbf{s}$. Hence,

$$
m_{l}\left(g, g^{\prime}\right)=\sum_{i=1}^{n} c^{\mathbf{t}_{i}}\left(g, g^{\prime}\right)
$$

such that a $\mathrm{GLAM}_{l}$ can be considered as a sum of GLCMs taken over these $n$ basic neighborhoods.

\subsubsection{Cardinal aura measure and aura matrix}

The above measure is adopted by most authors because it generalizes GLCMs and is well suited to image synthesis or processing problems $[6-16,26]$. However, this measure does not actually evaluate the number of sites of $S_{g^{\prime}}$ that are neighbors of any site of $S_{g}$, i.e., the number of sites that belong to $\mathcal{O}_{S_{g^{\prime}}}\left(S_{g}\right)$. We therefore also consider the most straightforward way to measure the size of an aura set, namely by its cardinal. We call it the cardinal aura measure and denote it as $m_{c}\left(g, g^{\prime}\right)$.

Definition 3. We define the cardinal aura measure of a gray-level set $S_{g}$ with respect to a set $S_{g^{\prime}}$ according to the neighborhood structure $\mathfrak{N}$ as:

$$
m_{c}\left(g, g^{\prime}\right)=\left|\mathcal{O}_{S_{g^{\prime}}}\left(S_{g}\right)\right| \stackrel{(1)}{=}\left|\bigcup_{\mathbf{s} \in S_{g}}\left(\mathcal{N}_{\mathbf{s}} \cap S_{g^{\prime}}\right)\right| .
$$

The corresponding cardinal GLAM $\left(\mathrm{GLAM}_{c}\right)$, denoted as $M_{c}$, is the $q \times q$ integer matrix defined by $M_{c}=\left[m_{c}\left(g, g^{\prime}\right)\right], 0 \leq g, g^{\prime} \leq q-1$.

\subsubsection{Numerical examples and interpretation}

Figure 1 is a numerical example for the above two aura measures. Figure 1(e) shows how to compute the local-count aura measure for $g=2, g^{\prime}=3$, and for the neighborhood defined at all sites $\mathbf{s}$ as shown in figure $1(\mathrm{~b})$ : the 9 arrows give $m_{l}(2,3)$. On figures $1(\mathrm{c})$ and $1(\mathrm{~d})$, we directly read the cardinal aura measures $m_{c}(2,3)=7$ as the number of boxes and $m_{c}(3,2)=5$ as the number of diamonds.

The local-count aura measure evaluates the number of times that a site of $S_{g^{\prime}}$ is a neighbor of a site of $S_{g}$, whereas the cardinal aura measure evaluates the number of sites of $S_{g^{\prime}}$ that are neighbors of a site of $S_{g}$. A high value for these two measures indicates that a lot of the sites of the two sets are close and/or mixed according to the neighborhood structure $\mathfrak{N}$. A low value indicates that $S_{g}$ and $S_{g^{\prime}}$ are rather separated from each other.

We retain both measures to compare the texture discriminative power of their respective aura matrices $M_{l}$ and $M_{c}$ used as texture descriptors. For example, the two aura matrices of the image 


\begin{tabular}{|l|l|l|l|l|l|l|l|}
\hline 0 & 0 & 5 & 5 & 0 & 0 & 5 & 5 \\
\hline 0 & 0 & 5 & 5 & 0 & 0 & 5 & 5 \\
\hline 0 & 0 & 5 & 5 & 0 & 0 & 5 & 5 \\
\hline 0 & 0 & 5 & 5 & 0 & 0 & 5 & 5 \\
\hline 0 & 0 & 5 & 5 & 0 & 0 & 5 & 5 \\
\hline 0 & 0 & 5 & 5 & 0 & 0 & 5 & 5 \\
\hline 0 & 0 & 5 & 5 & 0 & 0 & 5 & 5 \\
\hline 0 & 0 & 5 & 5 & 0 & 0 & 5 & 5 \\
\hline
\end{tabular}

(a) Texture image $I_{o}$

\begin{tabular}{|l|l|l|l|l|l|l|l|}
\hline 0 & 0 & 4 & 5 & 1 & 0 & 4 & 5 \\
\hline 0 & 0 & 4 & 5 & 1 & 0 & 4 & 5 \\
\hline 0 & 0 & 4 & 5 & 1 & 0 & 4 & 5 \\
\hline 0 & 0 & 4 & 5 & 1 & 0 & 4 & 5 \\
\hline 0 & 0 & 4 & 5 & 1 & 0 & 4 & 5 \\
\hline 0 & 0 & 4 & 5 & 1 & 0 & 4 & 5 \\
\hline 0 & 0 & 4 & 5 & 1 & 0 & 4 & 5 \\
\hline 0 & 0 & 4 & 5 & 1 & 0 & 4 & 5 \\
\hline
\end{tabular}

(b) Degraded texture image $I_{d}$

Figure 2: A synthetic gray-level texture image and its degraded version.

shown in figure 1(a) built with the neighborhood of figure 1(b) are:

$$
M_{l}=\left[\begin{array}{rrrr}
0 & 3 & 3 & 12 \\
3 & 0 & 0 & 0 \\
2 & 0 & 4 & 9 \\
13 & 0 & 9 & 2
\end{array}\right] \text { and } M_{c}=\left[\begin{array}{llll}
0 & 2 & 2 & 8 \\
3 & 0 & 0 & 0 \\
2 & 0 & 3 & 7 \\
8 & 0 & 5 & 2
\end{array}\right]
$$

Note that $m_{l}\left(g, g^{\prime}\right) \geq m_{c}\left(g, g^{\prime}\right)$ for any pair of gray levels $\left(g, g^{\prime}\right)[5]$, but all the cells that are zero in $M_{c}$ are also zero in $M_{l}$. However, the highest values do not occur exactly in the same cells: the cell $(0,3)$ contains the highest value of $M_{c}$ but not of $M_{l}$.

In the remainder of this paper, a gray-level aura matrix is simply called GLAM and denoted $M$ when the aura measure can be either of the above two, and similarly in the fuzzy case.

\subsection{Limitations of crisp aura}

Texture images always exhibit some degradations due to the imperfections in the image acquisition and encoding processes. In order to show the limitations of GLAMs to characterize such an imprecise texture information, let us consider a synthetic and simple texture image $I_{o}$ that represents four binary stripes (see figure 2(a)) and its degraded version $I_{d}$ (see figure $2(\mathrm{~b})$ ). Both are defined on a $8 \times 8$ lattice $\mathcal{S}$ and quantized with $q=6$ levels. Computing the GLAMs with the neighborhood of figure 1(b), we get for each image and each aura measure ${ }^{2}$ :

$$
M_{l}\left[I_{o}\right]=\left[\begin{array}{rrrrrr}
60 & 0 & 0 & 0 & 0 & 24 \\
0 & 0 & 0 & 0 & 0 & 0 \\
0 & 0 & 0 & 0 & 0 & 0 \\
0 & 0 & 0 & 0 & 0 & 0 \\
0 & 0 & 0 & 0 & 0 & 0 \\
24 & 0 & 0 & 0 & 0 & 60
\end{array}\right] \text { and } M_{l}\left[I_{d}\right]=\left[\begin{array}{rrrrrr}
37 & 8 & 0 & 0 & 16 & 0 \\
8 & 7 & 0 & 0 & 0 & 8 \\
0 & 0 & 0 & 0 & 0 & 0 \\
0 & 0 & 0 & 0 & 0 & 0 \\
16 & 0 & 0 & 0 & 14 & 16 \\
0 & 8 & 0 & 0 & 16 & 14
\end{array}\right]
$$

and:

$$
M_{c}\left[I_{o}\right]=\left[\begin{array}{rrrrrr}
32 & 0 & 0 & 0 & 0 & 24 \\
0 & 0 & 0 & 0 & 0 & 0 \\
0 & 0 & 0 & 0 & 0 & 0 \\
0 & 0 & 0 & 0 & 0 & 0 \\
0 & 0 & 0 & 0 & 0 & 0 \\
24 & 0 & 0 & 0 & 0 & 32
\end{array}\right] \text { and } M_{c}\left[I_{d}\right]=\left[\begin{array}{rrrrrr}
23 & 8 & 0 & 0 & 16 & 0 \\
8 & 7 & 0 & 0 & 0 & 8 \\
0 & 0 & 0 & 0 & 0 & 0 \\
0 & 0 & 0 & 0 & 0 & 0 \\
16 & 0 & 0 & 0 & 14 & 16 \\
0 & 8 & 0 & 0 & 16 & 14
\end{array}\right]
$$

\footnotetext{
${ }^{2}$ When required, like here, the source image is appended to a GLAM $M$ or a fuzzy GLAM $\tilde{M}$.
} 
Let us measure the similarity between these two images by the intersection between their normalized GLAMs $^{3}$, defined as (see section 3.3.4 for a detailed discussion):

$$
\operatorname{Inter}\left(M\left[I_{o}\right], M\left[I_{d}\right]\right)=\sum_{g=0}^{q-1} \sum_{g^{\prime}=0}^{q-1} \min \left(\frac{M\left[I_{o}\right]\left(g, g^{\prime}\right)}{\sum_{a=0}^{q-1} \sum_{a^{\prime}=0}^{q-1} M\left[I_{o}\right]\left(a, a^{\prime}\right)}, \frac{M\left[I_{d}\right]\left(g, g^{\prime}\right)}{\sum_{a=0}^{q-1} \sum_{a^{\prime}=0}^{q-1} M\left[I_{d}\right]\left(a, a^{\prime}\right)}\right) .
$$

The intersection between the texture image and its degraded version is either equal to $\operatorname{Inter}\left(M_{l}\left[I_{o}\right], M_{l}\left[I_{d}\right]\right)=$ 0.304 or to Inter $\left(M_{c}\left[I_{o}\right], M_{c}\left[I_{d}\right]\right)=0.240$, according to the aura measure used. Given that the intersection value ranges from 0 (for very different images) to 1 (very similar images), these low values show that GLAMs can be strongly affected by small changes of some gray levels. To avoid this problem, we propose to incorporate the notion of fuzzy sets in the aura concept.

\section{Fuzzy aura}

In this section, we first recall some notions about fuzzy sets and fuzzy numbers that are used to introduce fuzzy sets of a gray-level image. We then extend the aura concept to these so-called "fuzzy gray-level sets" and we explain how it is used to build fuzzy GLAMs (FGLAMs).

\subsection{Fuzzy gray-level set}

In the fuzzy set theory, the set $\mathcal{S}$ of all sites is called the universal set and a fuzzy subset $S$ of the universal set can be represented by a function $\mu_{S}: \mathcal{S} \rightarrow[0,1]$, called the membership function of $S$. The value $\mu_{S}(\mathbf{s})$ represents the membership degree of the site $\mathbf{s}$ to the set $S$. The ordinary subsets of $\mathcal{S}$, called crisp sets, can be considered as particular cases of its fuzzy subsets for which the membership degree is restricted to $\{0,1\}$. Given a gray-level image $I$ defined on $\mathcal{S}$ and quantized with $q$ levels, and a gray level $g, 0 \leq g \leq q-1$, we call fuzzy gray-level set the fuzzy set $S_{g} \subseteq \mathcal{S}$ defined by its membership function $\mu_{S_{g}}$ (i.e., by the membership degree $\mu_{S_{g}}(\mathbf{s})$ of each site $\left.\mathbf{s} \in \mathcal{S}\right)$.

To define $\mu_{S_{g}}$, we consider the gray levels as fuzzy numbers defined by Jawahar and Ray [20]. A fuzzy number $\tilde{g}$ is characterized by its membership function $\mu_{\tilde{g}}(x): \mathbb{R} \rightarrow[0,1]$ that can be defined in several ways [27]. It often takes the form $\mu_{\tilde{g}}(x)=f(|x-g|)$, where $f$ is a function which controls the shape of the fuzzy number and $g$ is the crisp counterpart of the fuzzy number $\tilde{g}[20,22]$. Some types of fuzzy numbers are particularly important, notably in applied fuzzy arithmetic [28]. Among them

\footnotetext{
${ }^{3}$ A GLAM $M$ is said to be normalized iff $\sum_{g, g^{\prime}} m\left(g, g^{\prime}\right)=1$. The normalization step ensures that two GLAMs are comparable even if the size of their source images are different or/and if the sum of all the cells in each GLAM differs, like for $M_{c}\left[I_{o}\right]$ and $M_{c}\left[I_{d}\right]$ in this example.
} 
are the symmetrical Gaussian and the triangular fuzzy numbers $\tilde{g}$ whose membership degrees of $x \in \mathbb{R}$ are:

$$
\mu_{\tilde{g}}(x)=\exp \left(-\frac{(x-g)^{2}}{2 \alpha^{2}}\right)
$$

and:

$$
\mu_{\tilde{g}}(x)=\max \left(0,1-\frac{|x-g|}{\alpha}\right),
$$

where $\alpha$ is a real positive constant which controls the span of the fuzzy number. The triangular fuzzy number is used for example by Jawahar and Ray for texture representation [20]. Its membership degree equals 1 at $x=g$, decreases as the difference between $x$ and $g$ increases, and cancels out when $|x-g| \geq \alpha$.

When fuzzy numbers are gray levels, the membership degree of each site $\mathbf{s}$ to the fuzzy set $S_{g}$ is the membership degree $\mu_{\tilde{g}}(I(\mathbf{s}))$ of the gray level $I(\mathbf{s})$ to the fuzzy number $\tilde{g}$ :

$$
\mu_{S_{g}}(\mathbf{s})=\mu_{\tilde{g}}(I(\mathbf{s}))
$$

where the discrete membership function $\mu_{\tilde{g}}:[0, \ldots, q-1] \rightarrow[0,1]$ defines the fuzzy number $\tilde{g}$.

\subsection{Fuzzy aura set}

\subsubsection{Definition}

In the fuzzy framework, the neighborhood $\mathcal{N}_{\mathbf{s}}$ of a site $\mathbf{s}$ is now a fuzzy subset of $\mathcal{S}$. The neighborhood of each site $\mathbf{s} \in \mathcal{S}$ is hence defined as a (membership) function of all the sites $\mathbf{r} \in \mathcal{S}$, hereafter referred to as the neighborhood function and denoted as $v_{\mathbf{s}}(\mathbf{r})$. In other words, $v_{\mathbf{s}}(\mathbf{r})$ is the membership degree of any site $\mathbf{r}$ to the neighborhood of a specific site $\mathbf{s}$.

Definition 4. Let $S_{g}$ and $S_{g^{\prime}}$ be two fuzzy gray-level sets defined by their membership functions $\mu_{S_{g}}$ and $\mu_{S_{g^{\prime}}}$. We define the fuzzy aura $\mathcal{O}_{S_{g^{\prime}}}\left(S_{g}\right)$ of $S_{g}$ with respect to $S_{g^{\prime}}$ as the fuzzy subset of $\mathcal{S}$ with the following membership degree at each site $\mathbf{r} \in \mathcal{S}$ :

$$
\mu_{\mathcal{O}_{S_{g^{\prime}}}\left(S_{g}\right)}(\mathbf{r})=\min \left\{\sup _{\mathbf{s} \in \mathcal{S}}\left[\min \left(\mu_{S_{g}}(\mathbf{s}), v_{\mathbf{s}}(\mathbf{r})\right)\right], \mu_{S_{g^{\prime}}}(\mathbf{r})\right\}
$$

JustificATion. The crisp aura set (see equation (1)) can be rewritten as:

$$
\mathcal{O}_{S_{g^{\prime}}}\left(S_{g}\right)=\bigcup_{\mathbf{s} \in \mathcal{S}}\left(\mathcal{N}_{\mathbf{s}, S_{g}} \cap S_{g^{\prime}}\right)
$$

where $\mathcal{N}_{\mathbf{s}, S_{g}}$ is the crisp set containing the neighboring sites $\mathbf{r}$ of each site $\mathbf{s} \in S_{g}: \mathcal{N}_{\mathbf{s}, S_{g}}=\left\{\mathbf{r} \in \mathcal{S},\left(\mathbf{s} \in S_{g}\right) \wedge\left(\mathbf{r} \in \mathcal{N}_{\mathbf{s}}\right)\right\}$. Its fuzzy counterpart is defined by the following membership degree at each site $\mathbf{r} \in \mathcal{S}$ :

$$
\mu_{\mathcal{N}_{\mathbf{s}, S_{g}}}(\mathbf{r})=\min \left(\mu_{S_{g}}(\mathbf{s}), v_{\mathbf{s}}(\mathbf{r})\right) .
$$




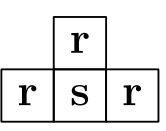

(a) $\mathcal{N}_{\mathbf{s}}$ (s: site of

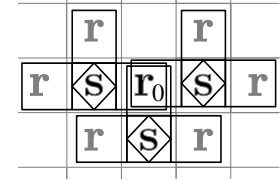

(b)

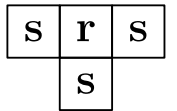

(c) $\check{\mathcal{N}}_{\mathbf{r}}(\mathbf{r}:$ site of

interest, $\mathbf{r}$ : neighbors

$\check{\mathcal{N}}_{\mathbf{r}_{0}}=\left\{\mathbf{s} \in \mathcal{S}, \mathbf{r}_{0} \in \mathcal{N}_{\mathbf{s}}\right\}$ interest, s: neighbors)

(diamonds)

Figure 3: Transposed neighborhood.

In this expression, the crisp logical operator and $(\wedge)$ is transcribed by the fuzzy operator min, and the membership of $\mathbf{r}$ to the neighborhood of $\mathbf{s}$ is transcribed by the degree $v_{\mathbf{s}}(\mathbf{r})$.

According to the fuzzy set theory [29], we define the fuzzy set $\mathcal{N}_{\mathbf{s}, S_{g}} \cap S_{g^{\prime}}$ of equation (10) by its membership function given by:

$$
\mu_{\mathcal{N}_{\mathbf{s}, S_{g}} \cap S_{g^{\prime}}}(\mathbf{r})=\min \left[\mu_{\mathcal{N}_{\mathbf{s}, S_{g}}}(\mathbf{r}), \mu_{S_{g^{\prime}}}(\mathbf{r})\right]
$$

The fuzzy aura set is defined by analogy with the crisp case. The crisp set union operator $\bigcup$ of equation (10) is transcribed by the fuzzy operator sup [29] to get the membership degree of the fuzzy aura set at each site $\mathbf{r} \in \mathcal{S}$ :

$$
\begin{aligned}
& \mu_{\mathcal{O}_{S_{g^{\prime}}}\left(S_{g}\right)}(\mathbf{r})=\sup _{\mathbf{s} \in \mathcal{S}}\left(\mu_{\mathcal{N}_{\mathbf{s}, S_{g}} \cap S_{g^{\prime}}}(\mathbf{r})\right) \\
& \stackrel{(12)}{=} \sup _{\mathbf{s} \in \mathcal{S}}\left\{\min \left[\mu_{\mathcal{N}_{\mathbf{s}, S_{g}}}(\mathbf{r}), \mu_{S_{g^{\prime}}}(\mathbf{r})\right]\right\} \\
&=\min \left\{\sup _{\mathbf{s} \in \mathcal{S}}\left[\mu_{\mathcal{N}_{\mathbf{s}, S_{g}}}(\mathbf{r})\right], \mu_{S_{g^{\prime}}}(\mathbf{r})\right\}
\end{aligned}
$$

Inserting the definition of $\mu_{\mathcal{N}_{\mathbf{s}, S_{g}}}(\mathbf{r})$ from equation (11) provides equation (9).

\subsubsection{Illustrative example}

To illustrate the computation of fuzzy aura sets according to equation (9), let us consider the neighborhood function $v_{\mathbf{s}}(\mathbf{r})$ defined identically at any site $\mathbf{s}$ as the crisp neighborhood $\mathcal{N}_{\mathbf{s}}$ of figure $1(\mathrm{~b})$ recalled in figure $3(\mathrm{a})$ :

$$
v_{\mathbf{s}}(\mathbf{r})= \begin{cases}1 & \text { if }\left(\|\mathbf{r}-\mathbf{s}\|_{1} \leq 1\right) \wedge\left(\mathbf{r}-\mathbf{s} \neq(0,1)^{\top}\right) \\ 0 & \text { otherwise }\end{cases}
$$

At each site $\mathbf{r}$, the sites $\mathbf{s}$ that satisfy $v_{\mathbf{s}}(\mathbf{r})=1$ belong to the neighborhood defined by the transposition of $\mathcal{N}_{\mathbf{s}}$, i.e., the neighborhood (usually denoted as $\check{\mathcal{N}}_{\mathbf{r}}$ ) reflected about the site of interest $\mathbf{r}$. This is illustrated in figure $3(\mathrm{~b})$ for a site $\mathbf{r}_{0}$ (the sites $\mathbf{s}$ of which $\mathbf{r}_{0}$ is a neighbor being shown as diamonds), and generalized to any site $\mathbf{r}$ in figure $3(\mathrm{c})$. 


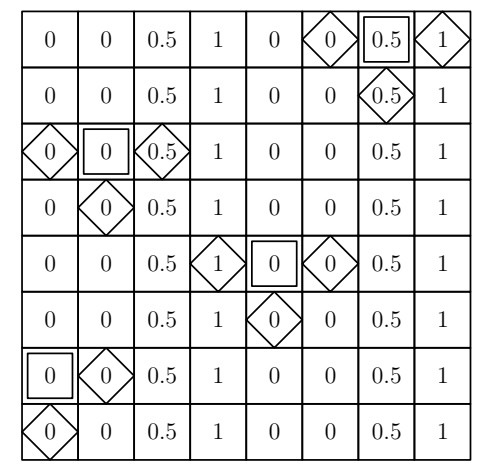

(a) Membership function $\mu_{S_{5}}$

\begin{tabular}{|c|c|c|c|c|c|c|c|}
\hline 0 & 0.5 & 0 & 0 & 0.5 & 0.5 & 0 & 0 \\
\hline 0 & 0.5 & 0 & 0 & 0.5 & 0.5 & 0 & 0 \\
\hline 0 & 0.5 & 0 & 0 & 0.5 & 0.5 & 0 & 0 \\
\hline 0 & 0.5 & 0 & 0 & 0.5 & 0.5 & 0 & 0 \\
\hline 0 & 0.5 & 0 & 0 & 0.5 & 0.5 & 0 & 0 \\
\hline 0 & 0.5 & 0 & 0 & 0.5 & 0.5 & 0 & 0 \\
\hline \hline 0 & 0.5 & 0 & 0 & 0.5 & 0.5 & 0 & 0 \\
\hline 0 & 0.5 & 0 & 0 & 0.5 & 0.5 & 0 & 0 \\
\hline
\end{tabular}

(d) Membership function $\mu_{\mathcal{O}_{S_{0}}}\left(S_{5}\right)$

\begin{tabular}{|c|c|c|c|c|c|c|c|}
\hline 0 & 0.5 & 1 & 1 & 1 & 0.5 & 1 & 1 \\
\hline 0 & 0.5 & 1 & 1 & 1 & 0.5 & 1 & 1 \\
\hline 0 & 0.5 & 1 & 1 & 1 & 0.5 & 1 & 1 \\
\hline 0 & 0.5 & 1 & 1 & 1 & 0.5 & 1 & 1 \\
\hline 0 & 0.5 & 1 & 1 & 1 & 0.5 & 1 & 1 \\
\hline 0 & 0.5 & 1 & 1 & 1 & 0.5 & 1 & 1 \\
\hline \hline 0 & 0.5 & 1 & 1 & 1 & 0.5 & 1 & 1 \\
\hline 0 & 0.5 & 1 & 1 & 1 & 0.5 & 1 & 1 \\
\hline
\end{tabular}

(b) $\sup _{\mathbf{s} \in \mathcal{S}}\left[\min \left(\mu_{S_{5}}(\mathbf{s}), v_{\mathbf{s}}(\mathbf{r})\right)\right]$

\begin{tabular}{|c|c|c|c|c|c|c|c|}
\hline 0 & 0 & 0.5 & 0 & 0 & 0 & 0.5 & 0 \\
\hline 0 & 0 & 0.5 & 0 & 0 & 0 & 0.5 & 0 \\
\hline 0 & 0 & 0.5 & 0 & 0 & 0 & 0.5 & 0 \\
\hline 0 & 0 & 0.5 & 0 & 0 & 0 & 0.5 & 0 \\
\hline 0 & 0 & 0.5 & 0 & 0 & 0 & 0.5 & 0 \\
\hline 0 & 0 & 0.5 & 0 & 0 & 0 & 0.5 & 0 \\
\hline \hline 0 & 0 & 0.5 & 0 & 0 & 0 & 0.5 & 0 \\
\hline 0 & 0 & 0.5 & 0 & 0 & 0 & 0.5 & 0 \\
\hline
\end{tabular}

(e) Membership function $\mu_{S_{3}}$

\begin{tabular}{|c|c|c|c|c|c|c|c|}
\hline 1 & 1 & 0 & 0 & 0.5 & 1 & 0 & 0 \\
\hline 1 & 1 & 0 & 0 & 0.5 & 1 & 0 & 0 \\
\hline 1 & 1 & 0 & 0 & 0.5 & 1 & 0 & 0 \\
\hline 1 & 1 & 0 & 0 & 0.5 & 1 & 0 & 0 \\
\hline 1 & 1 & 0 & 0 & 0.5 & 1 & 0 & 0 \\
\hline 1 & 1 & 0 & 0 & 0.5 & 1 & 0 & 0 \\
\hline \hline 1 & 1 & 0 & 0 & 0.5 & 1 & 0 & 0 \\
\hline 1 & 1 & 0 & 0 & 0.5 & 1 & 0 & 0 \\
\hline
\end{tabular}

(c) Membership function $\mu_{S_{0}}$

\begin{tabular}{|c|c|c|c|c|c|c|c|}
\hline 0 & 0 & 0.5 & 0 & 0 & 0 & 0.5 & 0 \\
\hline 0 & 0 & 0.5 & 0 & 0 & 0 & 0.5 & 0 \\
\hline 0 & 0 & 0.5 & 0 & 0 & 0 & 0.5 & 0 \\
\hline 0 & 0 & 0.5 & 0 & 0 & 0 & 0.5 & 0 \\
\hline 0 & 0 & 0.5 & 0 & 0 & 0 & 0.5 & 0 \\
\hline 0 & 0 & 0.5 & 0 & 0 & 0 & 0.5 & 0 \\
\hline \hline 0 & 0 & 0.5 & 0 & 0 & 0 & 0.5 & 0 \\
\hline \hline 0 & 0 & 0.5 & 0 & 0 & 0 & 0.5 & 0 \\
\hline
\end{tabular}

(f) Membership function $\mu_{\mathcal{O}_{S_{3}}}\left(S_{5}\right)$

Figure 4: Examples of fuzzy aura sets $\mathcal{O}_{S_{0}}\left(S_{5}\right)((\mathrm{a})-(\mathrm{d}))$ and $\mathcal{O}_{S_{3}}\left(S_{5}\right)((\mathrm{e})-(\mathrm{f}))$ computed on image $I_{d}$ of figure $2(\mathrm{~b})$ with the neighborhood function $v_{\mathbf{S}}(\mathbf{r})$ defined by equation (14). See text for box and diamond captions. 
Figure 4 shows two examples of fuzzy aura sets computed on the degraded synthetic texture image $I_{d}$ of figure 2(b) with the above neighborhood function. Figures 4(a)-4(d) illustrate the computation of $\mathcal{O}_{s_{0}}\left(S_{5}\right)$ as first example. Figures $4(\mathrm{a})$ and $4(\mathrm{c})$ show the membership degrees $\mu_{S_{5}}(\mathbf{s})$ and $\mu_{S_{0}}(\mathbf{s})$ of each site $\mathbf{s}$ to the fuzzy sets $S_{5}$ and $S_{0}$. They are defined according to equations (7) and (8) of the triangular membership function with $\alpha=2$. Equation (13) states that the membership degree of $\mathcal{O}_{s_{0}}\left(S_{5}\right)$ at each site $\mathbf{r}$ is the minimum between $\mu_{S_{0}}(\mathbf{r})$ and $\sup _{\mathbf{s} \in \mathcal{S}}\left[\mu_{\mathcal{N}_{\mathbf{s}, S_{5}}}(\mathbf{r})\right]$. Figure 4(b) shows $\sup _{\mathbf{s} \in \mathcal{S}}\left[\mu_{\mathcal{N}_{\mathbf{s}, S_{5}}}(\mathbf{r})\right]=\sup _{\mathbf{s} \in \mathcal{S}}\left[\min \left(\mu_{S_{5}}(\mathbf{s}), v_{\mathbf{s}}(\mathbf{r})\right)\right]$ for all $\mathbf{r} \in \mathcal{S}$. For four sample sites $\mathbf{r}$ shown as boxes in figure $4(\mathrm{a})$, the three sites $\mathbf{s}$ belonging to $\check{\mathcal{N}}_{\mathbf{r}}$ are shown as diamonds. At each site $\mathbf{r}$, the maximal of these three values is retained as $\sup _{\mathbf{s} \in \mathcal{S}}\left[\min \left(\mu_{S_{5}}(\mathbf{s}), v_{\mathbf{s}}(\mathbf{r})\right)\right]$ (simplified as $\sup _{\mathbf{s} \in \tilde{\mathcal{N}}_{\mathbf{r}}}\left[\mu_{S_{5}}(\mathbf{s})\right]$ since $v_{\mathbf{s}}(\mathbf{r})$ is binary), which provides the values of figure $4(\mathrm{~b})$. Note that $v_{\mathbf{s}}(\mathbf{r})$ is binary for simplicity purposes in this example but may more generally take any real value between 0 and 1 . At last, figure 4(d) gives the membership function of the fuzzy aura set $\mathcal{O}_{S_{0}}\left(S_{5}\right)$ computed as the site-wise minimum of figures 4 (b) and 4(c) (see equation (9)). The same four sample sites are shown as boxes in all figures to be easily located. This example shows that the membership degrees of sites whose gray levels are different from 0 and 5 (here, 1) can be non-zero in the fuzzy aura set $\mathcal{O}_{S_{0}}\left(S_{5}\right)$.

Figures $4(\mathrm{e})-4(\mathrm{f})$ illustrate the computation of $\mathcal{O}_{s_{3}}\left(S_{5}\right)$ as second example. Figure $4(\mathrm{e})$ shows that the membership function $\mu_{S_{3}}$ is not identically zero even if the gray level 3 is absent from the image, allowing $S_{3}$ to have a non-empty intersection with another fuzzy set. For instance, figure 4(f) shows that the fuzzy aura set $\mathcal{O}_{S_{3}}\left(S_{5}\right)$ is either not identically zero. This example illustrates that gray levels absent from the image (but close to existing ones) can contribute to a fuzzy aura set, which was not the case for crisp sets.

\subsubsection{Morphological formulation of a fuzzy aura set}

As in the crisp case (see equation (2)), when the neighborhood is defined identically at any site $\mathbf{s}$, the fuzzy aura set can also be defined using a morphological dilation. In the framework of fuzzy mathematical morphology, the structuring element is a fuzzy set defined by its membership function $v$ known as the structuring function. The most classical definition of the dilation of a fuzzy set $S_{g}$ by $v$ is at each site $\mathbf{s} \in \mathcal{S}[30]$ :

$$
D 2_{v}\left(\mu_{S_{g}}\right)(\mathbf{s})=\sup _{\mathbf{r} \in \mathcal{S}}\left[\min \left(\mu_{S_{g}}(\mathbf{r}), v(\mathbf{r}-\mathbf{s})\right)\right]
$$

Here, the structuring function $v$ depends on the translation vector $\mathbf{r}-\mathbf{s}$ and corresponds to the neighborhood function $v_{\mathbf{s}}(\mathbf{r})$ provided that the latter is spatially invariant. The fuzzy aura set can 
then be defined by transcribing the crisp morphological formulation (2) as:

$$
\mu_{\mathcal{O}_{S_{g^{\prime}}}\left(S_{g}\right)}(\mathbf{s})=\min \left\{\sup _{\mathbf{r} \in \mathcal{S}}\left[\min \left(\mu_{S_{g}}(\mathbf{r}), v_{\mathbf{s}}(\mathbf{r})\right)\right], \mu_{S_{g^{\prime}}}(\mathbf{s})\right\} \text {. }
$$

Swapping variables $\mathbf{r}$ and $\mathbf{s}$ we get:

$$
\mu_{\mathcal{O}_{S_{g^{\prime}}}\left(S_{g}\right)}(\mathbf{r})=\min \left\{\sup _{\mathbf{s} \in \mathcal{S}}\left[\min \left(\mu_{S_{g}}(\mathbf{s}), v_{\mathbf{r}}(\mathbf{s})\right)\right], \mu_{S_{g^{\prime}}}(\mathbf{r})\right\} .
$$

This morphological definition of a fuzzy aura set is fully consistent with equation (9) provided that $v_{\mathbf{r}}(\mathbf{s})$ identifies to $v_{\mathbf{s}}(\mathbf{r})$, i.e., that the neighborhood function is symmetric and spatially invariant.

\subsection{Fuzzy aura measures and matrices}

To characterize the spatial repartition of gray levels, we deduce two fuzzy aura measures of a fuzzy set $S_{g}$ with respect to a fuzzy set $S_{g^{\prime}}$ from their crisp counterparts (see section 2.2).

\subsubsection{Fuzzy local-count aura measure}

Definition 5. The fuzzy local-count aura measure $\tilde{m}_{l}\left(g, g^{\prime}\right)$ is defined as:

$$
\tilde{m}_{l}\left(g, g^{\prime}\right)=\sum_{\mathbf{r} \in \mathcal{S}} \sum_{\mathbf{s} \in \mathcal{S}} \min \left\{\min \left[\mu_{S_{g}}(\mathbf{s}), v_{\mathbf{s}}(\mathbf{r})\right], \mu_{S_{g^{\prime}}}(\mathbf{r})\right\}
$$

Note that in the case of a binary neighborhood function, each pair of sites $(\mathbf{r}, \mathbf{s})$ for which $v_{\mathbf{s}}(\mathbf{r})=1$ contributes to $\tilde{m}_{l}\left(g, g^{\prime}\right)$ by adding the minimum between $\mu_{S_{g}}(\mathbf{s})$ and $\mu_{S_{g^{\prime}}}(\mathbf{r})$.

Justification. Following the definition of $\mathcal{N}_{\mathbf{s}, S_{g}}$ (see equation (10)), the crisp local-count aura measure of $S_{g}$ with respect to $S_{g^{\prime}}$ given by equation (3) can be rewritten as $m_{l}\left(g, g^{\prime}\right)=\sum_{\mathbf{s} \in \mathcal{S}}\left|\mathcal{N}_{\mathbf{s}, S_{g}} \cap S_{g^{\prime}}\right|$. Given that the cardinal of a fuzzy set $S$ is $|S|=\sum_{\mathbf{r} \in \mathcal{S}} \mu_{S}(\mathbf{r})$ [29], the fuzzy local-count aura measure of the fuzzy gray-level set $S_{g}$ with respect to $S_{g^{\prime}}$ is defined by analogy with the crisp case as:

$$
\tilde{m}_{l}\left(g, g^{\prime}\right)=\sum_{\mathbf{s} \in \mathcal{S}} \sum_{\mathbf{r} \in \mathcal{S}} \mu_{\mathcal{N}_{\mathbf{s}, S g} \cap S_{g^{\prime}}}(\mathbf{r}) .
$$

The membership degree $\mu_{\mathcal{N}_{\mathbf{s}, S_{g}} \cap S_{g^{\prime}}}(\mathbf{r})$ is deduced from equations (11) and (12) as:

$$
\mu_{\mathcal{N}_{\mathbf{s}, S_{g}} \cap S_{g^{\prime}}}(\mathbf{r})=\min \left\{\min \left[\mu_{S_{g}}(\mathbf{s}), v_{\mathbf{s}}(\mathbf{r})\right], \mu_{S_{g^{\prime}}}(\mathbf{r})\right\}
$$

Inserting this expression into (16) yields equation (15) of the fuzzy local-count aura measure.

\subsubsection{Fuzzy cardinal aura measure}

Definition 6. The fuzzy cardinal aura measure $\tilde{m}_{c}\left(g, g^{\prime}\right)$ is exactly defined as in the crisp case (see equation (5)) and directly follows from equation (9):

$$
\tilde{m}_{c}\left(g, g^{\prime}\right)=\sum_{\mathbf{r} \in \mathcal{S}} \min \left\{\sup _{\mathbf{s} \in \mathcal{S}}\left[\min \left(\mu_{S_{g}}(\mathbf{s}), v_{\mathbf{s}}(\mathbf{r})\right)\right], \mu_{S_{g^{\prime}}}(\mathbf{r})\right\}
$$


given that $\tilde{m}_{c}\left(g, g^{\prime}\right)=\left|\mathcal{O}_{S_{g^{\prime}}}\left(S_{g}\right)\right|=\sum_{\mathbf{r} \in \mathcal{S}} \mu_{\mathcal{O}_{S_{g^{\prime}}}\left(S_{g}\right)}(\mathbf{r})$.

Note that this definition differs from that of $m_{l}$ (see equation (15)) because it uses a sup over $\mathbf{s}$ instead of a sum. In the case of a binary neighborhood function, for each site $\mathbf{r}$ we look for the sites $\mathbf{s}$ of which $\mathbf{r}$ is a neighbor $\left(v_{\mathbf{s}}(\mathbf{r})=1\right)$ and determine the maximal value of $\mu_{S_{g}}(\mathbf{s})$ among them. Then, the site $\mathbf{r}$ contributes to $m_{c}\left(g, g^{\prime}\right)$ by the minimum between this maximal value and its own membership degree $\mu_{S_{g^{\prime}}}(\mathbf{r})$ to $S_{g^{\prime}}$.

In the examples of figure 4 , the fuzzy local-count aura measures are $\tilde{m}_{l}(5,0)=12$ and $\tilde{m}_{l}(5,3)=15$. The fuzzy cardinal aura measures are easier to compute by hand from figures 4(d) and 4(f), and evaluate to $\tilde{m}_{c}(5,0)=\sum_{\mathbf{r} \in \mathcal{S}} \mu_{\mathcal{O}_{S_{0}}\left(S_{5}\right)}(\mathbf{r})=12$ and $\tilde{m}_{c}(5,3)=8$. As in the crisp case, these measures satisfy $\tilde{m}_{l}\left(g, g^{\prime}\right) \geq \tilde{m}_{c}\left(g, g^{\prime}\right)$ (see proof in appendix A).

\subsubsection{Fuzzy gray-level aura matrices}

Following the crisp scheme, we define a fuzzy aura matrix as a representation of the fuzzy aura measures for all the pairs of fuzzy sets in the lattice given by the gray level pairs $\left(g, g^{\prime}\right)$. However, we only consider some of the $q$ gray levels on which the image is quantized, namely the set $\mathcal{G}$ of $p$ levels such that $p \leq q$. The latter are the crisp counterparts of the retained fuzzy numbers, respectively denoted as $g$ and $\tilde{g}$ in equation (7). This allows us to reduce the size of FGLAMs used as texture descriptors.

Definition 7. The local-count FGLAM $\left(\mathrm{FGLAM}_{l}\right)$, denoted as $\tilde{M}_{l}$, is the $p \times p$ real matrix defined by $\tilde{M}_{l}=\left[\tilde{m}_{l}\left(g, g^{\prime}\right)\right],\left(g, g^{\prime}\right) \in \mathcal{G}^{2}$.

Note that as in the crisp case (see section 2.2.1), when the neighborhood function $v_{\mathbf{s}}(\mathbf{r})$ is binary and equals 1 for a single neighbor only, a $\mathrm{FGLAM}_{l}$ is identical to a fuzzy GLCM (FGLCM) (see proof in appendix B.1). More generally, for a neighborhood composed of $n$ neighbors, a $\mathrm{FGLAM}_{l}$ can be written as a sum of $n$ "basic" local-count FGLAMs provided that the neighborhood function is binary and only depends on $\mathbf{r}-\mathbf{s}$ (see proof in appendix B.2).

Definition 8. The cardinal FGLAM $\left(\mathrm{FGLAM}_{c}\right)$, denoted as $\tilde{M}_{c}$, is the $p \times p$ real matrix defined by $\tilde{M}_{c}=\left[\tilde{m}_{c}\left(g, g^{\prime}\right)\right],\left(g, g^{\prime}\right) \in \mathcal{G}^{2}$.

The FGLAMs of the texture image $I_{o}$ and the degraded texture image $I_{d}$ shown in figure 2, built with the neighborhood function of equation (14), are for each fuzzy aura measure:

$$
\tilde{M}_{l}\left[I_{o}\right]=\left[\begin{array}{rrrrrr}
60 & 30 & 0 & 0 & 12 & 24 \\
30 & 30 & 0 & 0 & 12 & 12 \\
0 & 0 & 0 & 0 & 0 & 0 \\
0 & 0 & 0 & 0 & 0 & 0 \\
12 & 12 & 0 & 0 & 30 & 30 \\
24 & 12 & 0 & 0 & 30 & 60
\end{array}\right] \text { and } \tilde{M}_{l}\left[I_{d}\right]=\left[\begin{array}{rrrrrr}
48.5 & 34 & 7.5 & 8 & 20 & 12 \\
34 & 33.5 & 7.5 & 8 & 12 & 16 \\
7.5 & 7.5 & 3.5 & 0 & 4 & 4 \\
8 & 8 & 0 & 7 & 15 & 15 \\
20 & 12 & 4 & 15 & 37 & 38 \\
12 & 16 & 4 & 15 & 38 & 37
\end{array}\right]
$$


and:

$$
\tilde{M}_{c}\left[I_{o}\right]=\left[\begin{array}{rrrrrr}
32 & 16 & 0 & 0 & 12 & 24 \\
16 & 16 & 0 & 0 & 12 & 12 \\
0 & 0 & 0 & 0 & 0 & 0 \\
0 & 0 & 0 & 0 & 0 & 0 \\
12 & 12 & 0 & 0 & 16 & 16 \\
24 & 12 & 0 & 0 & 16 & 32
\end{array}\right] \text { and } \tilde{M}_{c}\left[I_{d}\right]=\left[\begin{array}{rrrrrr}
27.5 & 20 & 4 & 8 & 20 & 12 \\
20 & 19.5 & 4 & 8 & 12 & 16 \\
7.5 & 7.5 & 3.5 & 0 & 4 & 4 \\
8 & 8 & 0 & 7 & 15 & 15 \\
20 & 12 & 4 & 8 & 23 & 24 \\
12 & 16 & 4 & 8 & 24 & 23
\end{array}\right]
$$

We see that some cells of these FGLAMs are non-zero although the corresponding levels are absent from the image, which was not the case in their crisp counterparts. For instance, $\tilde{M}_{l}\left[I_{d}\right](3,0)$ is 8 even if no site of $I_{d}$ has the level 3, whereas $M_{l}\left[I_{d}\right](3,0)$ equals 0 (see section 2.3). This is because when a gray level $g$ is absent from the image, the crisp set $S_{g}$ is empty and any co-occurrence with this level is zero. But the fuzzy set $S_{g}$ may be non-empty, as already noticed for $S_{3}$ in figure 4(e), allowing it (or $\mathcal{N}_{\mathbf{s}, S_{g}}$ ) to have a non-empty intersection with another fuzzy set.

\subsubsection{FGLAM-based similarity measure}

Like GLAMs, FGLAMs can be regarded as joint probability density functions provided they are normalized. Therefore, any of the measures considered by Rubner et al. [31] to compare density functions can be used as FGLAM-based similarity measure. Here, we retain the intersection [32] between FGLAMs that is one of the simplest similarity measures. Note that we have also tested other measures like the Jeffrey divergence, but without significant changes in classification results.

The similarity measure between two images $I_{1}$ and $I_{2}$ is given by the intersection between their normalized FGLAMs, which expresses as:

$$
\operatorname{Inter}\left(\tilde{M}\left[I_{1}\right], \tilde{M}\left[I_{2}\right]\right)=\sum_{\left(g, g^{\prime}\right) \in \mathcal{G}^{2}} \min \left(\frac{\tilde{M}\left[I_{1}\right]\left(g, g^{\prime}\right)}{\sum_{\left(a, a^{\prime}\right) \in \mathcal{G}^{2}} \tilde{M}\left[I_{1}\right]\left(a, a^{\prime}\right)}, \frac{\tilde{M}\left[I_{2}\right]\left(g, g^{\prime}\right)}{\sum_{\left(a, a^{\prime}\right) \in \mathcal{G}^{2}} \tilde{M}\left[I_{2}\right]\left(a, a^{\prime}\right)}\right) .
$$

When the two images share a similar spatial arrangement of gray levels, their similarity value is close to 1 . Although this does not necessarily mean that the two images contain the same texture, we assume so. On the other hand, a similarity measure value close to 0 means that the two textures are significantly different.

The similarity between the two example texture images $I_{o}$ and $I_{d}$ is evaluated from their FGLAMs (see previous section) as Inter $\left(\tilde{M}_{l}\left[I_{o}\right], \tilde{M}_{l}\left[I_{d}\right]\right)=0.726$ and Inter $\left(\tilde{M}_{c}\left[I_{o}\right], \tilde{M}_{c}\left[I_{d}\right]\right)=0.695$. These values are much higher than those measured between the normalized GLAMs (0.304 and 0.240), which suggests that FGLAMs are less affected by image degradation than GLAMs.

\subsubsection{Discussion}

The main contribution of fuzzy aura is a formalization of the relationships between neighboring sites thanks to the neighborhood function $v_{\mathbf{s}}(\mathbf{r})$ (see equations (9), (15) and (18)). This function may have 
any support size and shape, and may take any real value between 0 and 1 . Like classical neighborhoodbased approaches, we have considered in all examples that this function is spatially invariant. Under this condition, we have shown that the fuzzy aura set can be reformulated in fuzzy morphological terms when the neighborhood function is symmetric, and that the fuzzy local-count aura matrix can be considered as a generalization of a fuzzy co-occurrence matrix when the neighborhood function is binary.

However, designing an appropriate function $v_{\mathbf{s}}(\mathbf{r})$ for texture characterization is not easy because the fuzzy aura formalism is based on the comparison between the membership degrees to different fuzzy sets (namely, the membership degree $v_{\mathbf{s}}(\mathbf{r})$ of any site $\mathbf{r}$ to the neighborhood of a specific site $\mathbf{s}$, and the membership degree $\mu_{S_{g}}(\mathbf{s})$ of the site $\mathbf{s}$ to a fuzzy gray-level set $S_{g}$ ). To simplify its design, the neighborhood function $v_{\mathbf{s}}(\mathbf{r})$ is chosen binary in order to either fully or not at all take the contribution of each neighbor to the aura measure into account. Specifically, setting $v_{\mathbf{s}}(\mathbf{r})=0$ in equation (9) excludes the neighbor from $\mu_{\mathcal{O}_{S_{g^{\prime}}}\left(S_{g}\right)}$, while $v_{\mathbf{s}}(\mathbf{r})=1$ allows it to contribute to the fuzzy aura set through the membership degree of $\mathbf{s}$ to $S_{g}$. The contribution of neighbors of a site to the fuzzy aura measures hence only depends on membership degrees to fuzzy gray-level sets, which is more easy to apprehend.

We propose the following binary neighborhood function:

$$
v_{\mathbf{s}}(\mathbf{r})= \begin{cases}1 & \text { if } \mathbf{r} \in \mathcal{N}_{\mathbf{s}}, \\ 0 & \text { otherwise },\end{cases}
$$

$\mathcal{N}_{\mathbf{s}}$ being the crisp neighborhood centered at the site $\mathbf{s}$. The fuzzy aura measures defined by equations (15) and (18) then boil down to:

$$
\tilde{m}_{l}\left(g, g^{\prime}\right)=\sum_{\mathbf{s} \in \mathcal{S}} \sum_{\mathbf{r} \in \mathcal{N}_{\mathbf{s}}} \min \left(\mu_{S_{g}}(\mathbf{s}), \mu_{S_{g^{\prime}}}(\mathbf{r})\right)
$$

and

$$
\tilde{m}_{c}\left(g, g^{\prime}\right)=\sum_{\mathbf{r} \in \mathcal{S}} \min \left(\sup _{\mathbf{s} \in \tilde{\mathcal{N}}_{\mathbf{r}}}\left(\mu_{S_{g}}(\mathbf{s})\right), \mu_{S_{g^{\prime}}}(\mathbf{r})\right) .
$$

Note that the computation of $\tilde{m}_{l}\left(g, g^{\prime}\right)$ is based on the neighborhood $\mathcal{N}_{\mathbf{s}}$, whereas that of $\tilde{m}_{c}\left(g, g^{\prime}\right)$ is based on the transposed neighborhood $\check{\mathcal{N}}_{\mathbf{r}}$ (see figure 3 ).

As mentioned before, the above examples use a neighborhood function $v_{\mathbf{s}}(\mathbf{r})$ that is defined identically at any site $\mathbf{s}$ for better clarity. However, equations (9), (15) and (18) assume no such restriction about the spatial invariance of $v_{\mathbf{s}}(\mathbf{r})$. We now propose to consider a binary neighborhood function that is specific to each site, i.e., whose support size and shape can be different for each site.

\section{Spatially-variant neighborhood for CFA images}

In this section, we give a quick insight into when a spatially-variant neighborhood is useful and how it can be designed to build the FGLAM of an image. For this purpose, we focus on a neighborhood 


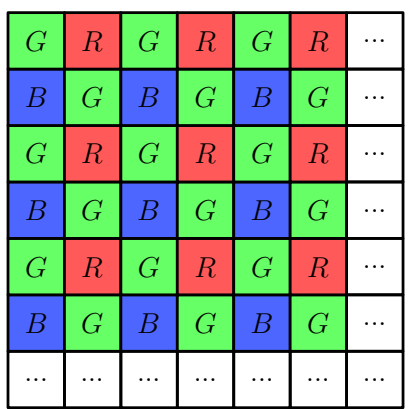

(a) $I_{C F A}$

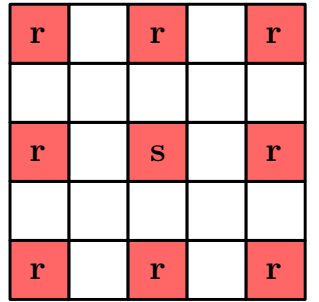

(b) $\mathcal{N}_{\mathbf{s}}^{R}, \mathbf{s} \in \mathcal{S}_{C F A}^{R}$

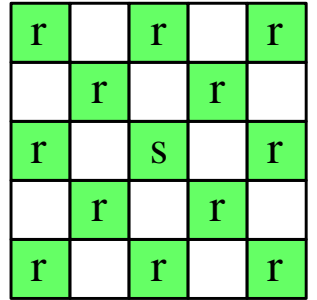

(c) $\mathcal{N}_{\mathbf{s}}^{G}, \mathbf{s} \in \mathcal{S}_{C F A}^{G}$

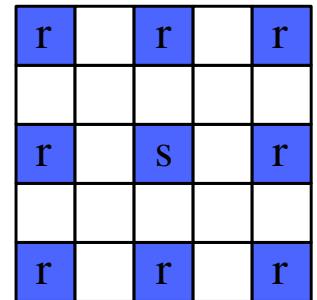

(d) $\mathcal{N}_{\mathbf{s}}^{B}, \mathbf{s} \in \mathcal{S}_{C F A}^{B}$

Figure 5: Spatially-variant neighborhood $\dot{\mathcal{N}}_{\mathbf{S}}((\mathrm{b})-(\mathrm{d})$ ) for the CFA image (a) (s: site of interest, $\mathbf{r}$ : neighbors, distance parameter: $d=2$ ).

dedicated to raw image description.

\subsection{Spatially-variant neighborhood}

To explicitly express the dependency of the neighborhood function $v_{\mathbf{s}}(\mathbf{r})$ on the site $\mathbf{s}$ while preserving notation consistency and compactness, we denote its spatially-variant version as $\dot{v}_{\mathbf{s}}(\mathbf{r})$ rather than $v_{\mathbf{s}}(\mathbf{r}, \mathbf{s})$. Given that a binary neighborhood function is considered, we propose the following design:

$$
\dot{v}_{\mathbf{s}}(\mathbf{r})= \begin{cases}1 & \text { if } \mathbf{r} \in \dot{\mathcal{N}}_{\mathbf{s}} \\ 0 & \text { otherwise }\end{cases}
$$

where $\dot{\mathcal{N}}_{\mathbf{s}}$ is the neighborhood considered at $\mathbf{s}$ and is specific to this site.

In this case, the fuzzy local-count aura measure of equation (15) is expressed just as in equation (21) as:

$$
\tilde{m}_{l}\left(g, g^{\prime}\right)=\sum_{\mathbf{s} \in \mathcal{S}} \sum_{\mathbf{r} \in \dot{\mathcal{N}}_{\mathbf{s}}} \min \left(\mu_{S_{g}}(\mathbf{s}), \mu_{S_{g^{\prime}}}(\mathbf{r})\right),
$$

but the fuzzy cardinal aura measure of equation (18) becomes:

$$
\tilde{m}_{c}\left(g, g^{\prime}\right)=\sum_{\mathbf{r} \in \mathcal{S}} \min \left(\sup _{\mathbf{s} \in \dot{\mathcal{X}}_{\mathbf{r}}}\left(\mu_{S_{g}}(\mathbf{s})\right), \mu_{S_{g^{\prime}}}(\mathbf{r})\right) .
$$

Here, $\dot{\mathcal{X}}_{\mathbf{r}}=\left\{\mathbf{x} \in \mathcal{S}, \mathbf{r} \in \dot{\mathcal{N}}_{\mathbf{x}}\right\}$ is the set of sites $\mathbf{x}$ whose own neighborhood $\dot{\mathcal{N}}_{\mathbf{x}}$ includes the site $\mathbf{r}$. Note that $\dot{\mathcal{X}}_{\mathbf{r}}$ is not a transposed neighborhood as $\check{\mathcal{N}}_{\mathbf{r}}$ in equation (21). Indeed, since the neighborhood function is specific to each site, the reciprocity of neighboring relationships between two sites is not guaranteed. In other words, when a site $\mathbf{r}$ belongs to the neighborhood $\dot{\mathcal{N}}_{\mathbf{s}}$ of a site $\mathbf{s}$, we cannot state that $\mathbf{s}$ belongs to the transposed neighborhood $\check{\mathcal{N}}_{\mathbf{r}}$ of $\mathbf{r}$ as in the case of a spatially-invariant neighborhood function (see section 3.2.2). Thus, the principle of the transposed neighborhood illustrated in figure 3 does not apply when the neighborhood function is spatially variant. 


\subsection{Neighborhood for CFA images}

Let us now consider an image analysis problem that highlights the usefulness of a spatially-variant neighborhood. Single-sensor color cameras deliver raw images, also known as color filter array (CFA) images, in which a single color component $k \in\{R, G, B\}$ is associated to each site according to its spatial coordinates [33]. The CFA image $I_{C F A}$ shown in figure $5($ a) stems from the widely-used Bayer CFA. It contains half of sites associated with the $G$ component arranged in a quincunx lattice and a quarter of sites associated with the $R$ or $B$ component arranged in a regular lattice. Each color component $k$ in $I_{C F A}$ is then defined on its own sublattice $\mathcal{S}_{C F A}^{k} \subset \mathcal{S}$ (see figures $5(\mathrm{~b})-(\mathrm{d})$ ).

To classify texture images acquired by a single-sensor color camera, one usually estimates the two color components missing at each site to form the color images. Losson et al. [33] show that this demosaicing step degrades the texture representation in the images and propose to extract six crisp chromatic co-occurrence matrices directly from CFA images. We follow the same line here but use a single fuzzy GLAM to characterize a CFA image.

For this purpose, we examine each sublattice $\mathcal{S}_{C F A}^{k}$ separately. To ensure that a site of interest $\mathbf{s}$ and its neighbors $\mathbf{r}$ are associated to the same color component, the considered neighborhood is therefore specifically designed according to the color component available at $\mathbf{s}$. We propose to define it as:

$$
\dot{\mathcal{N}}_{C F A, \mathbf{s}}= \begin{cases}\mathcal{N}_{C F A, \mathbf{s}}^{R} & \text { if } \mathbf{s} \in \mathcal{S}_{C F A}^{R}, \\ \mathcal{N}_{C F A, \mathbf{s}}^{G} & \text { if } \mathbf{s} \in \mathcal{S}_{C F A}^{G}, \\ \mathcal{N}_{C F A, \mathbf{s}}^{B} & \text { if } \mathbf{s} \in \mathcal{S}_{C F A}^{B},\end{cases}
$$

where the neighborhood $\mathcal{N}_{C F A, \mathbf{s}}^{k}$ for the color component $k$ is defined identically at any site $\mathbf{s} \in \mathcal{S}_{C F A}^{k}$ as $\mathcal{N}_{C F A, \mathbf{s}}^{k}=\left\{\mathbf{r} \in \mathcal{S}_{C F A}^{k},\left(d-1 \leq\|\mathbf{r}-\mathbf{s}\|_{\infty} \leq d\right) \wedge\left(\theta \bmod \frac{\pi}{4}=0\right)\right\}$ (see figures $\left.5(\mathrm{~b})-(\mathrm{d})\right)$. Here, $d$ is a distance parameter and $\theta$ denotes the angle between the horizontal unit vector and $\mathbf{r}-\mathbf{s}$. The considered neighbors are then:

- the 8 sites at infinity norm distance $d$ from $\mathbf{s}$ along the four main directions of the image plane (horizontal and vertical directions, and the two diagonals), when $R$ or $B$ is the color component available at $\mathbf{s}$ (see figures $5(\mathrm{~b})$ and $5(\mathrm{~d})$ );

- the 12 sites given as the previous 8 sites plus the 4 ones at distance $d-1$ from $\mathbf{s}$ along the two diagonals, when $G$ is available at $\mathbf{s}$ (see figure $5(\mathrm{c})$ ).

This allows us to define a spatially-variant neighborhood function (see equations (23) and (26)) suited to describe any CFA image by a single FGLAM. More explicitely, the local-count FGLAM of a CFA image gathers all the fuzzy local-count aura measures adapted from equation (24) as $\tilde{m}_{C F A, l}\left(g, g^{\prime}\right)=$ $\sum_{k \in\{R, G, B\}} \sum_{\mathbf{s} \in \mathcal{S}_{C F A}^{k}} \sum_{\mathbf{r} \in \dot{\mathcal{N}}_{C F A, \mathbf{s}}} \min \left(\mu_{S_{g}}(\mathbf{s}), \mu_{S_{g^{\prime}}}(\mathbf{r})\right)$, and similarly for the cardinal FGLAM from equation (25). 
The interest of such an approach for texture classification from CFA images is further investigated by experimental tests in the next section.

\section{Experiments and results}

In this section, we first quickly recall the parameters required to characterize a texture by the proposed approach and we detail how they are set up. Then we describe the used classification scheme and datasets. We finally present the classification results and a discussion follows.

\subsection{Texture characterization}

To characterize a texture by a FGLAM, we have to set several parameters. The first one is the number $p$ of fuzzy numbers $\tilde{g}$ that determine the size $p \times p$ of the FGLAM. The second parameter is the membership function $\mu_{\tilde{g}}$ that, at each site $\mathbf{s}$ of the image, defines the membership degree $\mu_{\tilde{g}}(I(\mathbf{s}))$ to any fuzzy number $\tilde{g}$ according to the site's gray level $I(\mathbf{s})$. At last, fuzzy aura measures are defined by the neighborhood function that models the spatial interactions between the sites.

\subsubsection{Number of fuzzy numbers}

Among the $q$ gray levels given by the image bit depth, we retain $p$ numbers that are the crisp counterparts of the considered fuzzy numbers. More precisely, we consider the $p$ numbers equally spaced between 0 and $q-1$ as the set $\mathcal{G}$. This equivalently defines $p$ disjoint intervals $[0, w-1],[w, 2 w-$ $1], \ldots,[(p-1) w, q-1]$ centered at these numbers, where $w=q / p$ is the interval width. As for $q, p$ is usually a power of 2 , so that $w$ is also an integer and a power of 2 . The numbers in $\mathcal{G}$ are the central values of these intervals (rounded to the ceiling integers), namely $\mathcal{G}=\left\{\left\lceil\frac{w-1}{2}\right\rceil,\left\lceil\frac{3 w-1}{2}\right\rceil, \ldots,\left\lceil q-\frac{w+1}{2}\right\rceil\right\}$.

We assess the classification accuracy achieved by FGLAMs with very low numbers of cells in order to evaluate how their memory cost can be reduced while preserving their relevance as texture descriptors. In practice, as the number $q$ of gray levels is equal to 256 , we set the number $p$ of intervals to 4 , 8 , and 16. The largest descriptors have then the same size $\left(p^{2}=256\right)$ as Local Binary Patterns (LBPs) that are one of the most efficient texture features [34]. Another motivation to retain low values of $p$ is that the difference between crisp and fuzzy aura increases when $p$ decreases, allowing us to examine the difference between crisp and fuzzy texture features. When $p$ decreases indeed, the interval associated to each fuzzy number is wider $(w=q / p)$ and the number of possible fuzzy membership degrees $\mu_{\tilde{g}}(I(\mathbf{s}))$ increases.

\subsubsection{Membership functions}

For each number $g \in \mathcal{G}$, the membership function $\mu_{\tilde{g}}:[0, q-1] \rightarrow[0,1]$ defines the associated fuzzy number $\tilde{g}$. This membership function should be maximal at $g$ and symmetric about $g$, but may 


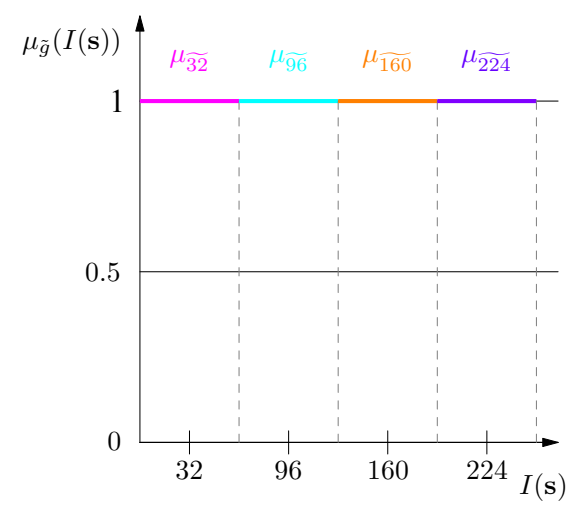

(a) Crisp

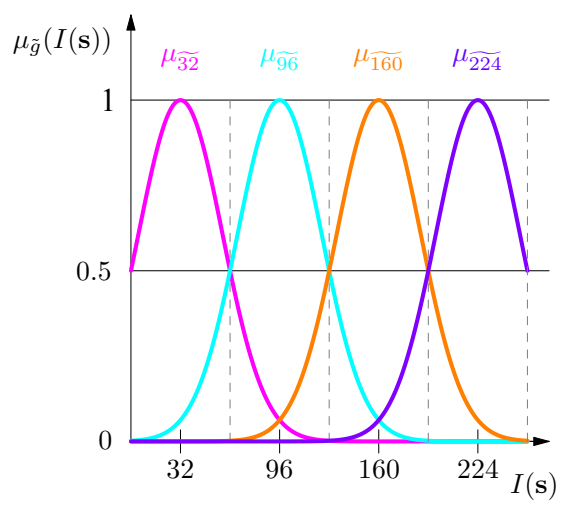

(b) Gaussian

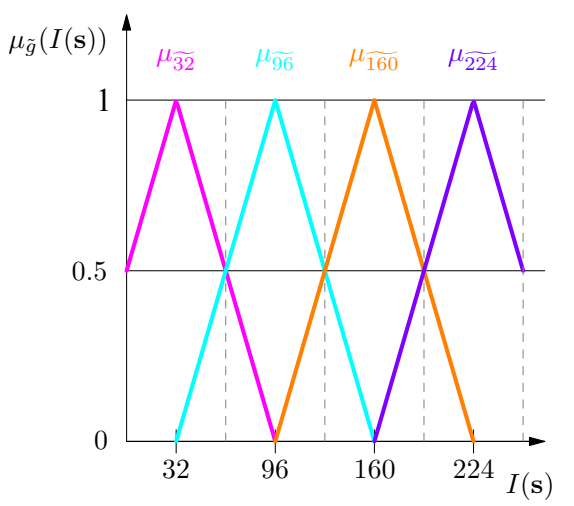

(c) Triangular

Figure 6: Membership functions $\mu_{\tilde{g}}, g \in \mathcal{G}$, for $p=4$ intervals when $q=256$.

be of any type and span. Figure 6 shows the three types of membership functions considered in our experiments: either crisp, Gaussian, or triangular. The latter two are defined by equations (6) with $\alpha=\frac{w}{2 \sqrt{2 \ln (2)}}$ and (7) with $\alpha=w$. They are designed to ensure that, for each interval centered at $g$, $\mu_{\tilde{g}}(I(\mathbf{s}))$ equals 0.5 at the interval bounds, i.e., for $I(\mathbf{s})=g \pm \frac{w}{2}$.

\subsubsection{Neighborhood functions}

When the neighborhood function is binary and invariant, equations (21) and (22) show that FGLAMs depend on the neighborhood $\mathcal{N}_{\mathbf{s}}$ defined identically at any site $\mathbf{s}$. We have also proposed to compute FGLAMs from a spatially-variant neighborhood $\dot{\mathcal{N}}_{\mathbf{s}}$ specifically defined at each site $\mathbf{s}$ (see equations (24) and (25)). $\dot{\mathcal{N}}_{\mathbf{s}}$ may depend on several parameters, like the distance between $\mathbf{s}$ and its neighbor $\mathbf{r}$, the difference between their levels or between their membership degrees to fuzzy numbers, or even a combination of these parameters.

For our experimental tests, we define the following spatially-invariant or site-specific neighborhood functions:

- $v$ (see equation (20)) that is based on the spatially-invariant neighborhood made of the 8 sites at distance $d$ from $\mathbf{s}$ along the four main directions of the image plane (horizontal and vertical directions, and the two diagonals $): \mathcal{N}_{\mathbf{s}}=\left\{\mathbf{r} \in \mathcal{S},\left(\|\mathbf{r}-\mathbf{s}\|_{\infty}=d\right) \wedge\left(\theta \bmod \frac{\pi}{4}=0\right)\right\}$.

- $\dot{v}^{\mu}$ (see equation (23)) that is based on the site-specific neighborhood made of only some of the latter 8 sites according to the rule $\dot{\mathcal{N}}_{\mathbf{s}}^{\mu}=\left\{\mathbf{r} \in \mathcal{S},\left(\mathbf{r} \in \mathcal{N}_{\mathbf{s}}\right) \wedge\left(\mu_{S_{g}}(\mathbf{s}) \geq \mu_{S_{g^{\prime}}}(\mathbf{r})\right)\right\}$. Among the 8 neighbors in $\mathcal{N}_{\mathbf{s}}$, we retain those whose membership degree to $S_{g^{\prime}}$ is lower than that of $\mathbf{s}$ to $S_{g}$, such that $\dot{\mathcal{N}}_{\mathbf{s}}^{\mu}$ is both specific to $\mathbf{s}$ and to the considered FGLAM cell $\left(g, g^{\prime}\right)$. The (strong) assumption for the final condition is that the membership degrees of $\mathbf{s}$ to $S_{g}$ and of $\mathbf{r}$ to $S_{g^{\prime}}$ remain in the same order when the gray levels change according to acquisition conditions. This 
is only true in the gray level intervals where the respective membership functions $\mu_{\tilde{g}}$ and $\mu_{\tilde{g}^{\prime}}$ are monotonic.

- $\dot{v}_{C F A}$ that is used to represent CFA images and is based on the site-specific neighborhood $\dot{\mathcal{N}}_{C F A, \mathbf{s}}$ of equation (26).

- $\dot{v}_{C F A}^{\mu}$ that is used to represent CFA images and is based on the site-specific neighborhood made of only some of the latter sites according to same final condition on the membership degrees as in $\dot{\mathcal{N}}_{\mathbf{s}}^{\mu}: \dot{\mathcal{N}}_{C F A, \mathbf{s}}^{\mu}=\left\{\mathbf{r} \in \mathcal{S},\left(\mathbf{r} \in \dot{\mathcal{N}}_{C F A, \mathbf{s}}\right) \wedge\left(\mu_{S_{g}}(\mathbf{s}) \geq \mu_{S_{g^{\prime}}}(\mathbf{r})\right)\right\}$.

\subsection{Texture classification}

\subsubsection{Global scheme and texture datasets}

Achieving supervised texture classification is to retrieve, among a set of train images grouped into texture classes, those that represent the same texture as a given test image. This matching scheme evaluates the similarity between the test image and each train image by a pairwise intersection between their respective descriptors according to equation (19). The same scheme also holds to compare two CFA images but for two color images, the similarity is the mean intersection of their three marginal FGLAMs $\tilde{M}^{k}$ computed on each color component $k=R, G, B$. The train images are then ranked with respect to their similarity to the test image, which provides the class of texture to which the test image belongs. We simply retain the class of the most similar train image according to the onenearest-neighbor $(1 \mathrm{NN})$ scheme, as it is usually done to assess the classification performance of new texture features $[4,32,33]$.

We perform extensive classification tests on Outex texture datasets [35], that are well known and widely used as benchmarks in the literature. Outex contains both gray-level and color images of a very large number of surface textures acquired with a 3-CCD camera under controlled conditions (see figure 7). However, we have found that the downloadable raster images of gray-level datasets provided biased results because of an intensity normalization. We have therefore built several new test sets from the original RGB images and made them available at http://lagis-vi univ-lille1.fr/datasets/outex.php. Outex-TC-00020-00023 (concisely denoted below as TC-20-23) are gray-level datasets generated from the same 68 color textures as in Outex-TC-00013 by using the intensity formula given in Outex description: $I=0.299 I^{R}+0.587 I^{G}+0.114 I^{B}$. Each dataset is characterized by a specific variation of acquisition conditions (see below), and Outex-TC-00030-00033 (TC-30-33) are the color counterparts.

Each dataset contains 68 classes made up of 40 images (or 160 images for TC-20 and -30) of size $128 \times 128$ pixels whose intensities or color components are represented with $q=256$ levels. To test the behavior of our descriptors against different degradations of the observed textures, we follow Outex's hold-out methodology and divide each dataset into: 

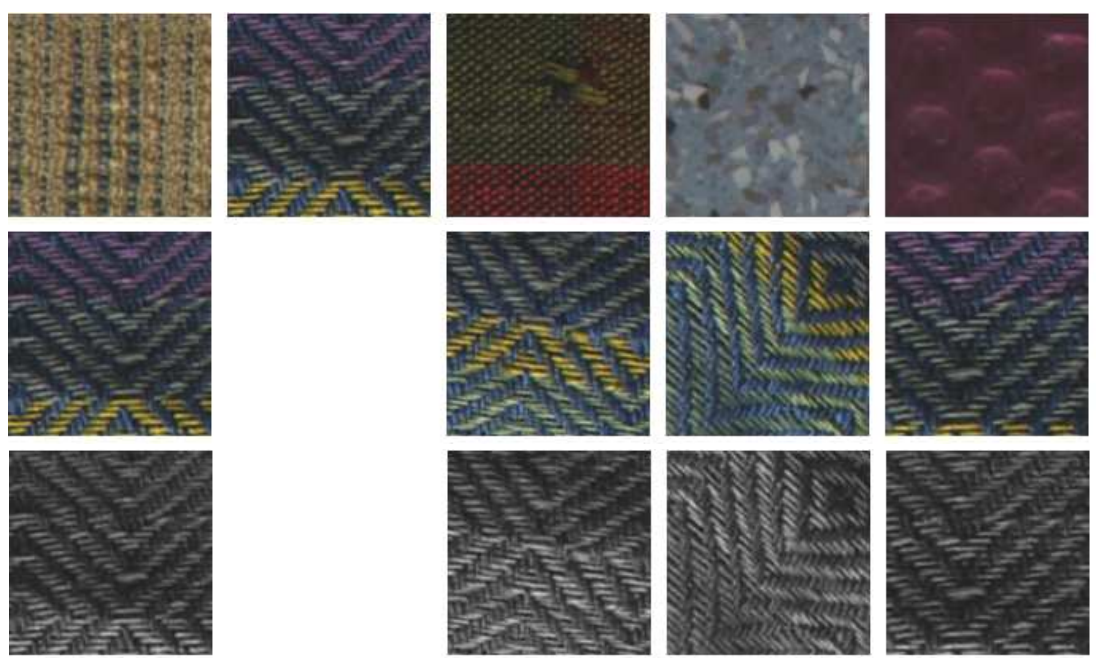
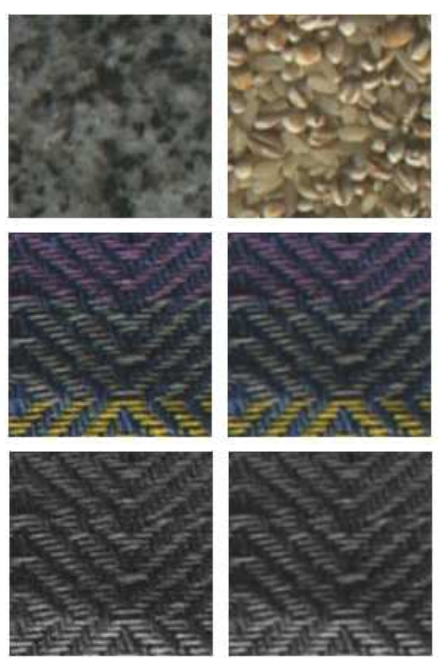

Figure 7: Examples of Outex textures from all the datasets used in our experiments. Top row: images of different classes (samples from TC-30-33 train images). Middle row: TC-30-33 train image of class "canvas023" acquired at 100 dpi under an incandescent light (left) and test images of the same class with different variations (from left to right: rotation angles of $10^{\circ}$ and $45^{\circ}$ (TC-30), 120 dpi resolution (TC-31), additive Gaussian noise with $\sigma=0.5$ (TC-32), Gaussian blur with $\rho=0.5$ (TC-33). Bottom row: gray-level counterparts (TC-20-23).

- the train subset that contains the same $68 \times 20=1,360$ images (either gray-level or color ones) for all the ten datasets. These images represent unrotated textures acquired under a $2,856 \mathrm{~K}$ incandescent light at a resolution of $100 \mathrm{dpi}$;

- the test subset, whose images represent the same textures as the train subset but acquired under varying conditions (see figure 7):

- TC-20 (gray-level) and -30 (color) contain 10, 880 test images rotated by 8 different angles $(5,10,15,30,45,60,75$, and 90 degrees);

- TC-21 and -31 contain 1, 360 test images acquired at a resolution of $120 \mathrm{dpi}$;

- TC-22 and -32 contain 1,360 test images corrupted by an additive Gaussian noise with a standard deviation $\sigma=5$;

- TC-23 and -33 contain 1, 360 test images blurred by a Gaussian filter with a radius of decay (standard deviation of the filter) $\rho=0.5$;

We also use the original Outex-TC-00013 (TC-13) color dataset and its gray-level version (TC-13-1), for which both the train and test subsets are made of half of the images.

The computation scheme of FGLAMs for gray-level, color, and CFA test images is outlined in figure 8. The computation of FGLAMs from train images follow the same outline but without any degradation. Because CFA images are unavailable in Outex, we simulate them from the original 


\section{Test image \\ formation by \\ degradation}

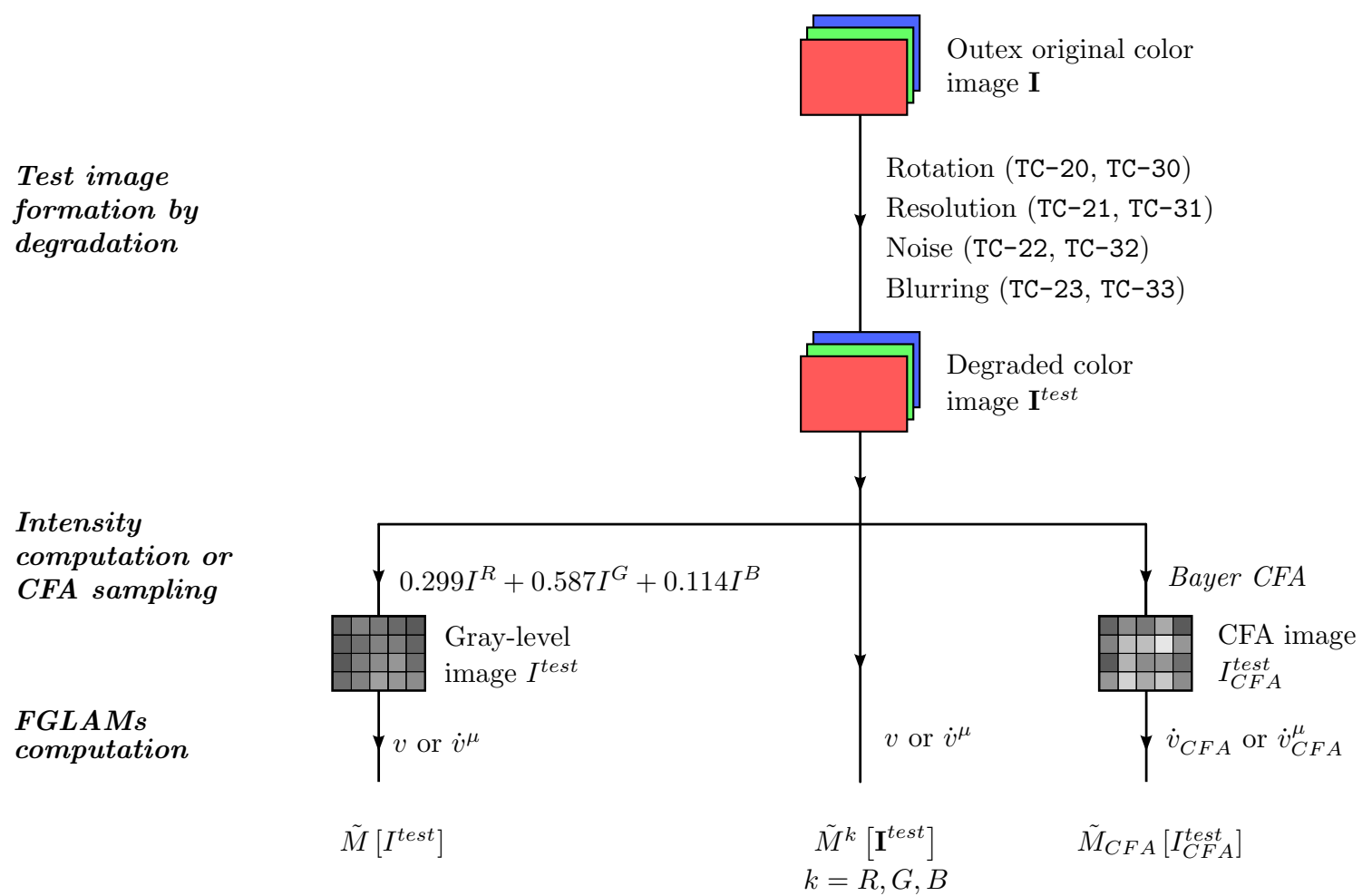

Figure 8: Outline of the computation of FGLAMs for different types of test images.

full-color images (train subset) or their degraded versions (test subset) by sampling a single color component at each pixel according to the Bayer CFA arrangement shown in figure 5(a).

\subsubsection{Classification results}

Tables 1-3 show the correct classification rates of the test images in all datasets for two values of the distance parameter $d$. The considered datasets are shown as main column headers and the secondary column headers contain the number $p$ of intervals associated to the fuzzy numbers. The row headers contain the major characteristics of the tested approaches. The two main row headers indicate the proposed FGLAMs $\left(\mathrm{FGLAM}_{l}\right.$ and $\left.\mathrm{FGLAM}_{c}\right)$ to compare their respective classification performances. For each FGLAM, three membership functions to the fuzzy numbers (crisp, Gaussian and triangular) are tested to assess the improvement brought by the fuzzy approach. The deepest row headers contain the two neighborhood functions considered for each FGLAM and a given membership function, according to the image type: FGLAMs of gray-level and of color images are computed with either $v$ or $\dot{v}^{\mu}$ to assess the interest of neighborhood variability, while FGLAMs of CFA images use either $\dot{v}_{C F A}$ or $\dot{v}_{C F A}^{\mu}$ to assess the interest of the membership-based neighbor selection $\left(\dot{\mathcal{N}}_{C F A, \mathbf{s}}^{\mu}\right)$ in a spatially-variant neighborhood $\left(\dot{\mathcal{N}}_{C F A, \mathbf{s}}\right)$. Note that we compute crisp FGLAMs only with the neighborhood function $v$ (or $\dot{v}_{C F A}$ ) to stick with the crisp framework (recall that $\dot{v}^{\mu}$ and $\dot{v}_{C F A}^{\mu}$ use a neighbor selection based on membership degrees to fuzzy numbers). The values written in bold 
Table 1: Correct classification rates (\%) on Outex gray-level datasets.

(a) $d=2$

\begin{tabular}{|c|c|c|c|c|c|c|c|c|c|c|c|c|c|c|c|c|c|}
\hline & \multirow{2}{*}{$\begin{array}{l}\text { Membership } \\
\text { function }\end{array}$} & \multirow{2}{*}{$\begin{array}{l}\text { Neighborhood } \\
\text { function }\end{array}$} & \multicolumn{3}{|c|}{ TC-13-1 } & \multicolumn{3}{|c|}{$\mathrm{TC}-20$} & \multicolumn{3}{|c|}{$\mathrm{TC}-21$} & \multicolumn{3}{|c|}{ TC-22 } & \multicolumn{3}{|c|}{$\mathrm{TC}-23$} \\
\hline & & & $p=4$ & $p=8$ & $p=16$ & $p=4$ & $p=8$ & $p=16$ & $p=4$ & $p=8$ & $p=16$ & $p=4$ & $p=8$ & $p=16$ & $p=4$ & $p=8$ & $p=16$ \\
\hline \multirow{5}{*}{ 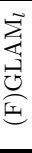 } & Crisp & $v$ & 46.03 & 58.97 & 76.62 & 47.47 & 61.96 & 79.33 & 45.88 & 59.78 & 78.82 & 38.68 & 46.99 & 54.85 & 49.04 & 67.57 & 89.93 \\
\hline & \multirow{2}{*}{ Gaussian } & $v$ & 64.71 & 68.82 & 73.38 & 65.00 & 70.97 & 75.79 & 66.69 & 69.63 & 74.78 & 62.57 & 62.87 & 64.12 & 89.85 & 92.13 & 93.75 \\
\hline & & $\dot{v}^{\mu}$ & 69.56 & 71.76 & 75.59 & 70.53 & 73.93 & 77.69 & 71.18 & 72.79 & 75.74 & 60.00 & 63.97 & 65.07 & 89.56 & 93.01 & 94.41 \\
\hline & \multirow{2}{*}{ Triangular } & $v$ & 59.56 & 70.88 & 78.53 & 57.79 & 71.40 & 79.84 & 56.76 & 69.93 & 78.01 & 57.65 & 64.41 & 61.18 & 85.07 & 90.81 & 95.22 \\
\hline & & $\dot{v}^{\mu}$ & 68.38 & 73.68 & 79.41 & 68.34 & 75.18 & 80.88 & 67.21 & 74.04 & 78.97 & 64.41 & 63.97 & 60.29 & 89.71 & 93.60 & 95.66 \\
\hline \multirow{5}{*}{ 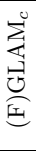 } & Crisp & $v$ & 48.24 & 63.09 & 77.94 & 49.17 & 65.04 & 81.34 & 48.97 & 65.37 & 79.41 & 41.32 & 51.03 & 56.40 & 52.35 & 68.16 & 90.74 \\
\hline & \multirow{2}{*}{ Gaussian } & $v$ & 70.15 & 73.68 & 76.91 & 70.27 & 75.00 & 78.80 & 69.78 & 75.96 & 77.35 & 54.34 & 56.69 & 58.60 & 86.84 & 88.24 & 90.37 \\
\hline & & $\dot{v}^{\mu}$ & 74.71 & 76.18 & 78.24 & 75.29 & 77.99 & 80.03 & 76.69 & 78.46 & 77.79 & 52.13 & 54.56 & 57.72 & 85.29 & 89.12 & 91.76 \\
\hline & \multirow{2}{*}{ Triangular } & $v$ & 64.85 & 75.44 & 79.71 & 65.70 & 75.47 & 82.16 & 65.44 & 74.49 & 80.00 & 54.34 & 55.15 & 57.35 & 80.74 & 85.66 & 91.03 \\
\hline & & $\dot{v}^{\mu}$ & 72.50 & 76.76 & 80.15 & 73.57 & 78.61 & 82.51 & 75.37 & 76.47 & 80.07 & 52.21 & 53.31 & 56.69 & 82.65 & 87.06 & 92.43 \\
\hline
\end{tabular}

(b) $d=4$

\begin{tabular}{|c|c|c|c|c|c|c|c|c|c|c|c|c|c|c|c|c|c|}
\hline & \multirow{2}{*}{$\begin{array}{l}\text { Membership } \\
\text { function }\end{array}$} & \multirow{2}{*}{$\begin{array}{l}\text { Neighborhood } \\
\text { function }\end{array}$} & \multicolumn{3}{|c|}{ TC-13-1 } & \multicolumn{3}{|c|}{ TC-20 } & \multicolumn{3}{|c|}{$\mathrm{TC}-21$} & \multicolumn{3}{|c|}{$\mathrm{TC}-22$} & \multicolumn{3}{|c|}{$\mathrm{TC}-23$} \\
\hline & & & $p=4$ & $p=8$ & $p=16$ & $p=4$ & $p=8$ & $p=16$ & $p=4$ & $p=8$ & $p=16$ & $p=4$ & $p=8$ & $p=16$ & $p=4$ & $p=8$ & $p=16$ \\
\hline \multirow{5}{*}{ 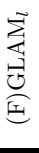 } & Crisp & $v$ & 38.09 & 56.62 & 75.15 & 40.64 & 56.99 & 77.10 & 37.65 & 56.62 & 74.71 & 43.53 & 53.01 & 61.03 & 48.60 & 68.60 & 91.69 \\
\hline & \multirow{2}{*}{ Gaussian } & $v$ & 63.82 & 67.35 & 71.62 & 63.30 & 69.09 & 74.19 & 63.24 & 67.65 & 71.10 & 63.01 & 64.49 & 64.71 & 91.47 & 92.50 & 95.00 \\
\hline & & $\dot{v}^{\mu}$ & 64.41 & 69.71 & 73.82 & 66.66 & 71.43 & 76.02 & 67.21 & 67.94 & 72.94 & 60.88 & 64.93 & 64.12 & 89.78 & 93.16 & 94.78 \\
\hline & \multirow{2}{*}{ Triangular } & $v$ & 59.12 & 70.15 & 76.62 & 56.31 & 69.90 & 78.58 & 53.75 & 67.50 & 76.18 & 62.28 & 65.00 & 61.32 & 89.19 & 92.57 & 95.88 \\
\hline & & $\dot{v}^{\mu}$ & 66.32 & 71.32 & 77.79 & 66.54 & 73.31 & 79.65 & 64.56 & 70.37 & 77.35 & 64.78 & 64.63 & 62.35 & 91.10 & 94.63 & 95.66 \\
\hline \multirow{5}{*}{ 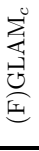 } & Crisp & $v$ & 45.29 & 61.62 & 77.79 & 43.94 & 61.00 & 79.36 & 45.07 & 60.29 & 78.09 & 47.13 & 53.46 & 59.85 & 54.71 & 71.47 & 92.72 \\
\hline & \multirow{2}{*}{ Gaussian } & $v$ & 72.21 & 73.82 & 77.21 & 70.20 & 74.54 & 78.19 & 69.34 & 74.78 & 75.96 & 58.53 & 59.04 & 60.37 & 88.90 & 90.07 & 92.50 \\
\hline & & $\dot{v}^{\mu}$ & 73.97 & 76.32 & 77.79 & 73.02 & 76.37 & 78.72 & 71.40 & 75.29 & 75.74 & 58.68 & 57.57 & 58.90 & 88.90 & 91.10 & 92.79 \\
\hline & \multirow{2}{*}{ Triangular } & $v$ & 66.32 & 75.00 & 78.97 & 65.03 & 75.03 & 81.19 & 62.43 & 73.46 & 79.71 & 58.31 & 56.40 & 58.75 & 84.93 & 88.68 & 93.16 \\
\hline & & $\dot{v}^{\mu}$ & 71.62 & 75.44 & 80.00 & 71.63 & 76.77 & 81.21 & 69.93 & 75.22 & 78.68 & 59.04 & 54.85 & 58.16 & 86.25 & 90.37 & 94.12 \\
\hline
\end{tabular}

Table 2: Correct classification rates (\%) on Outex color datasets.

(a) $d=2$

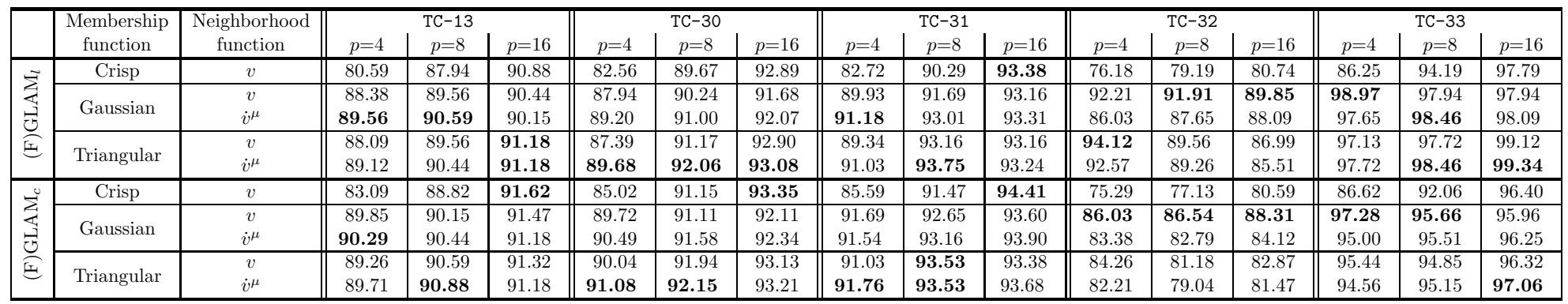

(b) $d=4$

\begin{tabular}{|c|c|c|c|c|c|c|c|c|c|c|c|c|c|c|c|c|c|}
\hline & \multirow{2}{*}{$\begin{array}{l}\text { Membership } \\
\text { function }\end{array}$} & \multirow{2}{*}{$\begin{array}{l}\text { Neighborhood } \\
\text { function }\end{array}$} & \multicolumn{3}{|c|}{$\mathrm{TC}-13$} & \multicolumn{3}{|c|}{$\mathrm{TC}-30$} & \multicolumn{3}{|c|}{$\mathrm{TC}-31$} & \multicolumn{3}{|c|}{$\mathrm{TC}-32$} & \multicolumn{3}{|c|}{ TC-33 } \\
\hline & & & $p=4$ & $p=8$ & $p=16$ & $p=4$ & $p=8$ & $p=16$ & $p=4$ & $p=8$ & $p=16$ & $p=4$ & $p=8$ & $p=16$ & $p=4$ & $p=8$ & $p=16$ \\
\hline \multirow{5}{*}{ 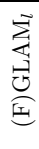 } & Crisp & $v$ & 78.53 & 87.94 & 90.59 & 80.97 & 89.09 & 92.74 & 80.96 & 90.22 & 93.16 & 78.09 & 80.96 & 82.94 & 87.21 & 95.07 & 97.94 \\
\hline & \multirow{2}{*}{ Gaussian } & $v$ & 87.79 & 88.82 & 89.85 & 87.85 & 89.96 & 91.39 & 89.71 & 90.29 & 91.84 & 91.69 & 91.69 & 89.56 & 99.12 & 98.16 & 98.75 \\
\hline & & $\dot{v}^{\mu}$ & 88.09 & 89.26 & 90.44 & 89.06 & 90.97 & 91.87 & 89.71 & 91.10 & 92.50 & 86.84 & 87.43 & 87.65 & 97.65 & 98.90 & 98.97 \\
\hline & \multirow{2}{*}{ Triangular } & $v$ & 87.35 & 89.41 & 90.74 & 87.56 & 91.21 & 92.59 & 88.46 & 92.06 & 93.31 & 94.78 & 89.34 & 87.21 & 97.65 & 98.75 & 99.49 \\
\hline & & $\dot{v}^{\mu}$ & 88.97 & 89.71 & 91.32 & 89.54 & 91.71 & 92.93 & 89.04 & 92.13 & 93.46 & 91.03 & 89.34 & 86.10 & 97.87 & 99.49 & 99.56 \\
\hline \multirow{5}{*}{ 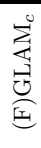 } & Crisp & $v$ & 80.74 & 87.06 & 90.88 & 82.99 & 90.51 & 93.19 & 83.01 & 91.32 & 93.82 & 77.13 & 78.09 & 82.65 & 86.62 & 92.57 & 98.31 \\
\hline & \multirow{2}{*}{ Gaussian } & $v$ & 90.15 & 90.00 & 90.88 & 89.60 & 91.21 & 92.04 & 90.88 & 92.72 & 93.75 & 87.57 & 87.94 & 88.60 & 97.35 & 96.47 & 97.28 \\
\hline & & $\dot{v}^{\mu}$ & 90.29 & 90.15 & 90.44 & 90.22 & 91.49 & 92.26 & 91.84 & 93.01 & 93.90 & 85.96 & 86.47 & 86.32 & 96.47 & 97.06 & 97.50 \\
\hline & \multirow{2}{*}{ Triangular } & $v$ & 88.09 & 90.15 & 91.32 & 89.88 & 91.90 & 92.90 & 91.69 & 93.46 & 93.68 & 86.84 & 84.71 & 84.71 & 95.81 & 96.32 & 98.01 \\
\hline & & $\dot{v}^{\mu}$ & 89.56 & 90.29 & 90.44 & 90.75 & 92.10 & 93.06 & 91.99 & 93.75 & 93.75 & 84.78 & 82.50 & 83.97 & 95.59 & 97.43 & 98.68 \\
\hline
\end{tabular}


Table 3: Correct classification rates (\%) on CFA images of Outex color datasets.

(a) $d=2$

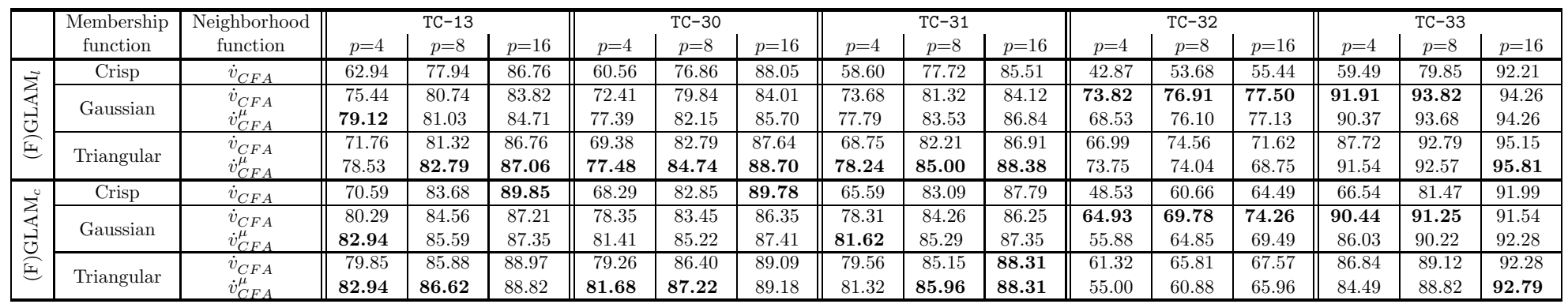

(b) $d=4$

\begin{tabular}{|c|c|c|c|c|c|c|c|c|c|c|c|c|c|c|c|c|c|}
\hline & \multirow{2}{*}{$\begin{array}{l}\text { Membership } \\
\text { function }\end{array}$} & \multirow{2}{*}{$\begin{array}{l}\text { Neighborhood } \\
\text { function }\end{array}$} & \multicolumn{3}{|c|}{$\mathrm{TC}-13$} & \multicolumn{3}{|c|}{$\mathrm{TC}-30$} & \multicolumn{3}{|c|}{$\mathrm{TC}-31$} & \multicolumn{3}{|c|}{ TC-32 } & \multicolumn{3}{|c|}{$\mathrm{TC}-33$} \\
\hline & & & $p=4$ & $p=8$ & $p=16$ & $p=4$ & $p=8$ & $p=16$ & $p=4$ & $p=8$ & $p=16$ & $p=4$ & $p=8$ & $p=16$ & $p=4$ & $p=8$ & $p=16$ \\
\hline \multirow{5}{*}{ 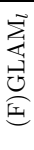 } & Crisp & $\dot{v}_{C F A}$ & 58.53 & 77.65 & 86.76 & 58.66 & 76.39 & 88.24 & 58.46 & 76.47 & 86.10 & 55.37 & 65.59 & 68.01 & 65.66 & 83.97 & 94.93 \\
\hline & \multirow{2}{*}{ Gaussian } & $\dot{v}_{C F A}$ & 74.26 & 80.44 & 84.56 & 72.42 & 79.81 & 84.35 & 73.16 & 80.07 & 84.04 & 76.76 & 78.31 & 79.34 & 93.31 & 93.53 & 95.15 \\
\hline & & $\dot{v}_{C F A}^{\mu}$ & 77.06 & 80.00 & 84.56 & 75.72 & 82.06 & 85.74 & 77.35 & 81.91 & 85.22 & 73.46 & 78.90 & 77.13 & 91.25 & 94.26 & 95.59 \\
\hline & \multirow{2}{*}{ Triangular } & $\dot{v}_{C F A}$ & 71.76 & 81.91 & 86.18 & 70.51 & 82.95 & 87.62 & 69.41 & 82.65 & 86.99 & 72.65 & 77.57 & 75.44 & 89.85 & 93.38 & 96.25 \\
\hline & & $\dot{v}_{C F A}^{\mu}$ & 77.21 & 82.79 & 87.35 & 76.23 & 84.67 & 88.65 & 75.37 & 83.75 & 88.53 & 76.91 & 76.62 & 73.53 & 91.40 & 94.56 & 97.50 \\
\hline \multirow{5}{*}{ 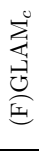 } & Crisp & $\dot{v}_{C F A}$ & 68.38 & 81.91 & 89.85 & 65.85 & 81.76 & 89.68 & 64.71 & 82.57 & 90.22 & 58.53 & 67.43 & 70.59 & 71.03 & 82.87 & 95.15 \\
\hline & \multirow{2}{*}{ Gaussian } & $\dot{v}_{C F A}$ & 81.76 & 85.15 & 87.50 & 78.75 & 83.87 & 86.80 & 79.49 & 84.63 & 87.35 & 70.66 & 73.97 & 77.50 & 91.47 & 91.99 & 93.68 \\
\hline & & $\dot{v}_{C F A}^{\mu}$ & 82.21 & 86.62 & 88.24 & 80.74 & 85.08 & 87.60 & 80.37 & 86.25 & 88.46 & 69.26 & 70.88 & 74.19 & 89.34 & 91.69 & 93.46 \\
\hline & \multirow{2}{*}{ Triangular } & $\dot{v}_{C F A}$ & 79.56 & 87.94 & 89.56 & 79.56 & 86.83 & 89.53 & 79.12 & 85.88 & 89.56 & 68.53 & 69.26 & 72.79 & 88.38 & 91.84 & 94.63 \\
\hline & & $\dot{v}_{C F A}^{\mu}$ & 82.35 & 87.65 & 89.26 & 81.38 & 87.29 & 89.45 & 81.40 & 86.69 & 89.85 & 66.25 & 68.16 & 70.74 & 87.43 & 91.99 & 95.07 \\
\hline
\end{tabular}

Table 4: Comparison of the approaches for the same cases as in (a) and (b) of tables 1-3: $d \in\{2,4\}$ and $p \in\{4,8,16\}$

\begin{tabular}{|c|c|c|c|c|c|c|c|c|c|c|c|c|c|c|c|c|c|c|}
\hline \multirow{2}{*}{$\begin{array}{ll}\text { Compared } \\
\text { approaches }\end{array}$} & \multicolumn{5}{|c|}{ Gray-level } & \multicolumn{5}{|c|}{ Color } & \multicolumn{5}{|c|}{ CFA } & \multirow{2}{*}{ Total } & \multicolumn{2}{|c|}{ Number of cases in } \\
\hline & $\mathrm{TC}-13-1$ & $\mathrm{TC}-20$ & $\mathrm{TC}-21$ & $\mathrm{TC}-22$ & $\mathrm{TC}-23$ & $\mathrm{TC}-13$ & $\mathrm{TC}-30$ & $\mathrm{TC}-31$ & $\mathrm{TC}-32$ & $\mathrm{TC}-33$ & $\mathrm{TC}-13$ & $\mathrm{TC}-30$ & $\mathrm{TC}-31$ & $\mathrm{TC}-32$ & $\mathrm{TC}-33$ & & dataset & total \\
\hline$\overline{F G L A M}_{c}>$ FGLAM $_{l}$ & 30 & 30 & 30 & 5 & 6 & 25 & 30 & 29 & 0 & 2 & 30 & 30 & 29 & 6 & 4 & 286 & 30 & 450 \\
\hline with $v$ or $\dot{v}_{C F A}$ & 18 & 18 & 18 & 5 & 6 & 17 & 18 & 18 & 0 & 2 & 18 & 18 & 18 & 6 & 4 & 184 & 18 & 270 \\
\hline with $\dot{v}^{\mu}$ or $\dot{v}_{C F A}^{\mu}$ & 12 & 12 & 12 & 0 & 0 & 8 & 12 & 11 & 0 & 0 & 12 & 12 & 11 & 0 & 0 & 102 & 12 & 180 \\
\hline FGLAM $>$ GLAM & 20 & 20 & 19 & 23 & 22 & 19 & 16 & 17 & 24 & 20 & 16 & 16 & 19 & 24 & 21 & 296 & 24 & 360 \\
\hline Gaussian $>$ Crisp & 8 & 8 & 8 & 12 & 10 & 8 & 7 & 8 & 12 & 10 & 8 & 8 & 8 & 12 & 10 & 137 & 12 & 180 \\
\hline Triangular $>$ Crisp & 12 & 12 & 11 & 11 & 12 & 11 & 9 & 9 & 12 & 10 & 8 & 8 & 11 & 12 & 11 & 159 & 12 & 180 \\
\hline $\begin{array}{l}\dot{v}^{\mu}>v \\
\dot{v}_{C F A}^{\mu}>\dot{v}_{C F A}\end{array}$ & 24 & 24 & 22 & 8 & 18 & 18 & 24 & 21 & 0 & 17 & 19 & 23 & 23 & 3 & 11 & $\begin{array}{c}176 \\
79\end{array}$ & $\begin{array}{l}24 \\
24\end{array}$ & $\begin{array}{l}240 \\
120\end{array}$ \\
\hline Triangular > Gaussian & 16 & 16 & 11 & 6 & 13 & 12 & 22 & 13 & 6 & 13 & 18 & 22 & 19 & 2 & 12 & 201 & 24 & 360 \\
\hline
\end{tabular}


face indicate which pair of membership and neighborhood functions provides the best classification accuracy for each value of $p$, each type of FGLAM, and each dataset.

A cell-wise comparison of tables 1-3 shows that the classification results obtained with gray-level images (see table 1) are lower than those obtained with color images (see table 2), and CFA images provide intermediate results ${ }^{4}$ (see table 3 ). This was expected because taking the color information into account generally increases the texture classification performance [4]. CFA images also carry color information due to their underlying color pattern, but the spatial definition of each color component $k$ is twice $(k=G)$ or four times $(k=R, B)$ lower than that of the corresponding full-color images. Note that the two values of the distance parameter $d$ generally provide close results, and that none of them overall exhibits a clear advantage.

We propose to examine these tables to answer three questions: Is the new cardinal aura measure interesting with regard to the original local-count one? Does the fuzzy aura measure provide better results than the crisp one? Does the neighborhood spatial adaptivity bring any improvement? We hence interpret the results in tables 1-3 according to three general comparisons that are summarized in table 4:

- Cardinal vs. local-count fuzzy aura measure $\left(\mathrm{FGLAM}_{c}\right.$ vs. $\left.\mathrm{FGLAM}_{l}\right)$ : the accuracy provided by the $\mathrm{FGLAM}_{c}$ is higher than that provided by the $\mathrm{FGLAM}_{l}$ computed with the same parameters ("FGLAM ${ }_{c}>$ FGLAM $_{l}$ " in table 4) in 286 out of the 450 cases (63.6\%). This global result splits up according to the neighborhood function as 184 out of the 270 cases (68.1\%) with $v$ or $\dot{v}_{C F A}$, and in 102 out of the 180 cases $(56.7 \%)$ with $\dot{v}^{\mu}$ or $\dot{v}_{C F A}^{\mu}$. We conclude that the aura set cardinal generally better characterizes the textures than the aura measure proposed by Elfadel and Picard, notably with spatially-invariant neighborhoods and almost only for the datasets whose test images undergo no acquisition condition variations (TC-13-1, TC-13) or variations in rotation (TC-20, TC-30) or scale (TC-21, TC-31).

- Crisp vs. fuzzy approach (GLAM vs. FGLAM): when the same neighborhood function $v$ (or $\left.\dot{v}_{C F A}\right)$ is used at each site, we can compare the classification performance of the crisp and fuzzy (Gaussian or triangular) membership functions. Over all datasets, a better classification accuracy is obtained by a fuzzy GLAM ("FGLAM > GLAM" in table 4) in 296 out of the 360 cases $(82.2 \%)$. Such superiority holds for all image types and all degradations. In addition, the FGLAMs computed with a Gaussian membership function yield better results than GLAMs in 137 out of the 180 cases $(76.1 \%)$, and in 159 cases (88.3\%) when the triangular membership function is used. These results show that representing the gray-level sets by fuzzy sets improves

\footnotetext{
${ }^{4}$ Except for 3 cases out of 300, for which gray-level accuracies are slightly higher than CFA ones, all being above $94 \%$.
} 
the classification performances because small local variations of the gray levels are better taken into account.

For the TC-13-1 dataset, FGLAMs provide $23.2 \%$ better classification rates on average than crisp matrices when $p=4$, but only $12.8 \%$ average better rates when $p=8$ and $0.4 \%$ when $p=16$. These average differences reach $36.3 \%, 16.0 \%$ and $2.2 \%$ for TC-23 dataset. The same trend can be observed for all the other datasets, and is particularly outstanding with gray-level and CFA images. Over all the datasets, a fuzzy matrix provides better results than the crisp one in all but one case ${ }^{5}$ when $p=4$ or 8 , but in only about half of the cases when $p=16$. When $p$ increases indeed, the width $w$ of the interval associated to each fuzzy number decreases, as well as its number of possible membership degrees. The classification results therefore confirm that the larger the number of intervals, the more FGLAMs are similar to GLAMs.

- Spatially-invariant vs. site-specific neighborhood function $\left(v\right.$ vs. $\left.\dot{v}^{\mu}\right)$ : in 176 out of the 240 cases (73.3\%), FGLAMs provide higher accuracy when computed with the site-specific neighborhood function $\dot{v}^{\mu}$ than when computed with the spatially-invariant neighborhood function $v$ (" $\dot{v}^{\mu}>v$ " in table 4). Except for noisy images (TC-22, TC-32), the neighborhood adaptivity based on membership degrees to fuzzy level sets is therefore an interesting approach to describe textures. The same result holds to a lesser extent for CFA images, for which the location- and membershipbased adaptive neighborhood function $\dot{v}_{C F A}^{\mu}$ provides better results than the sole location-based one $\dot{v}_{C F A}$ in 79 out of the 120 cases $(65.8 \%)$.

Let us also recall that local-count FGLAMs can be considered as FGLCMs when the neighborhood function is binary and spatially-invariant, as reported in section 3.3.3. To assess the performance of each approach, we pairwise compare the classification rates obtained for $v$ and $\dot{v}^{\mu}$ neighborhood functions for the sole $\mathrm{FGLAM}_{l}$ descriptor, for each combination of the other parameters (each value of $p$ and $d$, and either Gaussian or triangular membership function). Tables $1-3$ show that $\dot{v}^{\mu}$ provides better classification rates than $v$ in 137 of the 180 cases $(76.1 \%)$. This result confirms the benefit of the aura approach with regard to the co-occurrence one in the fuzzy framework because the fuzzy aura copes with spatially-variant neighborhoods.

Note at last that the triangular membership function provides better results than the Gaussian one in 201 out of the 360 cases (55.8\%) (see bottom row of table 4). Hence, we retain it in the discussion below together with the spatially-variant neighborhood function $\dot{v}^{\mu}$.

\footnotetext{
${ }^{5}$ TC-30 color dataset, $d=2, p=8$, FGLAM $_{c}$
} 


\subsection{Discussion}

\subsubsection{Comparison with LBP-like operators}

The performance of FGLAMs in texture classification can be compared with that of other classical texture descriptors. Among them, LBP histograms are known to be particularly powerful features because LBPs are invariant to any monotonic transformation of the gray levels and they are computed efficiently $[34,36]$. They have been extended into many variants that exhibit extra interesting properties like rotational invariance or multi-scale/temporal dimension/color handling.

In [37], Guo et al. investigate the discriminative power of the classical LBP based on the sign $(S)$ component, and propose to complete it with the center gray level $(C)$ and the magnitude $(M)$ components to form a so-called Completed LBP (CLBP). These three components can be considered separately or combined pairwise in either a joint or a concatenated histogram. This provides descriptors of various sizes, depending on whether the three components are taken separately, or jointly, or else hybridly (the joint histogram of two components is concatenated with that of the third one). Joint histograms are often prohibitively large, unless uniform patterns ${ }^{6}$ (denoted by the $u 2$ superscript) are considered for the $S$ and $M$ components. Moreover, texture rotation can be effectively handled by the rotationinvariant versions (superscripted as ri) of the components. The authors of [37] test various CLBP component combinations and other texture features, and show that the 3D joint histogram denoted as CLBP_S $S_{P, R}^{r i u 2} / M_{P, R}^{r i u 2} / C$ yields the best classification results among all the tested descriptors.

We compare the results of FGLAMs whose size is $l=p^{2}$ with various CLBP descriptors of similar sizes given by the number $P$ of neighbors (uniform patterns can take $P+2$ distinct values) and the combination of components (either the sum or the product of the component sizes, depending on whether they are concatenated or considered jointly). Specifically, for each value of $d \in\{2,4\}$ and of $p \in\{4,8,16\}$, the better among $\mathrm{FGLAM}_{l}\left(M_{l}\right)$ and $\mathrm{FGLAM}_{c}\left(M_{c}\right)$ descriptors computed with the triangular membership function and the spatially-variant neighborhood function $\dot{v}^{\mu}$ is compared to the best CLBP descriptor of similar size $l$ computed with the same number of neighbors $P=8$ and the same distance $R=d$ according to:

- for $p=4, \mathrm{M}_{l}$ and $\mathrm{M}_{c}(l=16)$ vs. $\mathrm{CLBP}_{-} S_{8, d}^{r i u 2}(l=10), \mathrm{CLBP}_{-} M_{8, d}^{\text {riu2 }}(l=10)$, and CLBP_S $S_{8, d}^{r i u 2} \_M_{8, d}^{r i u 2}(l=20)$;

- for $p=8, \mathrm{M}_{l}$ and $\mathrm{M}_{c}(l=64)$ vs. CLBP_$S_{8, d}^{r i u 2}{ }_{-} M_{8, d}^{r i u 2} / C(l=30)$, CLBP $\_M_{8, d}^{r i u 2}{ }_{-} S_{8, d}^{r i u 2} / C$ $(l=30)$, and CLBP_S $S_{8, d}^{r i u 2} / M_{8, d}^{r i u 2}(l=100)$;

\footnotetext{
6 "Uniform" LBPs refer to the patterns for which the number of bitwise $0 / 1$ changes in the circular binary presentation of the $P$ neighbors is 2 or less.
} 
Table 5: Classification performance of FGLAMs vs. other descriptors. FGLAMs are computed with a triangular membership function and the spatially-variant neighborhood function $\dot{v}^{\mu}$, and all descriptors use a 8-neighborhood at distance $d$.

(a) FGLAMs vs. LBPs on Outex gray-level datasets (associated variations are recalled in parentheses)

\begin{tabular}{|c|c|c|c|c|c|c|c|c|c|c|c|c|c|c|c|c|}
\hline \multirow[b]{2}{*}{$d$} & \multirow{2}{*}{$\begin{array}{c}\text { Descriptor } \\
\text { type }\end{array}$} & \multicolumn{3}{|c|}{$\overline{T C-13-1}$} & \multicolumn{3}{|c|}{ TC-20 (rotation) } & \multicolumn{3}{|c|}{$\mathrm{TC}-21$ (resolution) } & \multicolumn{3}{|c|}{ TC-22 (noise) } & \multicolumn{3}{|c|}{ TC-23 (blur) } \\
\hline & & $p=4$ & $p=8$ & $p=16$ & $p=4$ & $p=8$ & $p=16$ & $p=4$ & $p=8$ & $p=16$ & $p=4$ & $p=8$ & $p=16$ & $p=4$ & $p=8$ & $p=16$ \\
\hline \multirow[b]{2}{*}{2} & FGLAM & $\begin{array}{c}72.50 \\
M_{c}\end{array}$ & $\begin{array}{c}76.76 \\
M_{c}\end{array}$ & $\begin{array}{c}80.15 \\
M_{c}\end{array}$ & $\begin{array}{c}73.57 \\
M_{c}\end{array}$ & $\begin{array}{c}78.61 \\
M_{c}\end{array}$ & $\begin{array}{c}82.51 \\
M_{c}\end{array}$ & $\begin{array}{r}75.37 \\
M_{c}\end{array}$ & $\begin{array}{c}76.47 \\
M_{c}\end{array}$ & $\begin{array}{c}80.07 \\
M_{c}\end{array}$ & $\begin{array}{c}64.41 \\
M_{l}\end{array}$ & $\begin{array}{c}63.97 \\
M_{l}\end{array}$ & $\begin{array}{c}60.29 \\
M_{l}\end{array}$ & $\begin{array}{c}89.71 \\
M_{l}\end{array}$ & $\begin{array}{c}93.60 \\
M_{l}\end{array}$ & $\begin{array}{c}95.66 \\
M_{l}\end{array}$ \\
\hline & LBP & $\begin{array}{c}74.71 \\
S \_M\end{array}$ & $\begin{array}{r}81.91 \\
S / M \\
\end{array}$ & $\begin{array}{l}83.38 \\
S / M / C\end{array}$ & $\begin{array}{c}69.10 \\
S \\
\end{array}$ & $\begin{array}{l}55.49 \\
S / M\end{array}$ & $\begin{array}{l}85.78 \\
S / M / C\end{array}$ & $\begin{array}{l}56.47 \\
S_{-} M \\
\end{array}$ & $\begin{array}{r}90.51 \\
S / M\end{array}$ & $\begin{array}{c}82.35 \\
S\end{array}$ & $\begin{array}{c}19.34 \\
S\end{array}$ & $\begin{array}{l}33.68 \\
S / M \\
\end{array}$ & $\begin{array}{c}19.34 \\
S\end{array}$ & $\begin{array}{l}49.26 \\
S \_M \\
\end{array}$ & $\begin{array}{c}98.97 \\
S / M \\
\end{array}$ & $\begin{array}{c}93.75 \\
S \\
\end{array}$ \\
\hline \multirow[b]{2}{*}{4} & FGLAM & $\begin{array}{c}71.62 \\
M_{c}\end{array}$ & $\begin{array}{c}75.44 \\
M_{c}\end{array}$ & $\begin{array}{c}80.00 \\
M_{c}\end{array}$ & $\begin{array}{c}71.63 \\
M_{c}\end{array}$ & $\begin{array}{c}76.77 \\
M_{c}\end{array}$ & $\begin{array}{c}81.21 \\
M_{c}\end{array}$ & $\begin{array}{c}69.93 \\
M_{c}\end{array}$ & $\begin{array}{c}75.22 \\
M_{c}\end{array}$ & $\begin{array}{c}78.68 \\
M_{c}\end{array}$ & $\begin{array}{c}64.78 \\
M_{l}\end{array}$ & $\begin{array}{c}64.63 \\
M_{l}\end{array}$ & $\begin{array}{c}62.35 \\
M_{l}\end{array}$ & $\begin{array}{c}91.10 \\
M_{l}\end{array}$ & $\begin{array}{c}94.63 \\
M_{l}\end{array}$ & $\begin{array}{c}95.66 \\
M_{l}\end{array}$ \\
\hline & LBP & $\begin{array}{r}71.62 \\
S_{-} M\end{array}$ & $\begin{array}{r}76.91 \\
S / M\end{array}$ & $\begin{array}{c}76.91 \\
S / M / C\end{array}$ & $\begin{array}{r}72.96 \\
S_{-} M\end{array}$ & $\begin{array}{l}53.55 \\
S / M\end{array}$ & $\begin{array}{c}79.48 \\
S / M / C\end{array}$ & $\begin{array}{l}60.59 \\
S_{-} M\end{array}$ & $\begin{array}{r}93.24 \\
S / M\end{array}$ & $\begin{array}{c}78.16 \\
S\end{array}$ & $\begin{array}{l}27.50 \\
S_{-} M\end{array}$ & $\begin{array}{l}56.62 \\
S / M\end{array}$ & $\begin{array}{c}49.12 \\
S\end{array}$ & $\begin{array}{l}70.07 \\
S \_M\end{array}$ & $\begin{array}{c}100.00 \\
S / M\end{array}$ & $\begin{array}{c}100.00 \\
S\end{array}$ \\
\hline
\end{tabular}

(b) FGLAMs vs. LBPs on Outex color datasets

\begin{tabular}{|c|c|c|c|c|c|c|c|c|c|c|c|c|c|c|c|c|}
\hline \multirow[b]{2}{*}{$d$} & \multirow{2}{*}{$\begin{array}{l}\text { Descriptor } \\
\text { type }\end{array}$} & \multicolumn{3}{|c|}{$\mathrm{TC}-13$} & \multicolumn{3}{|c|}{$\mathrm{TC}-30$ (rotation) } & \multicolumn{3}{|c|}{ TC-31 (resolution) } & \multicolumn{3}{|c|}{ TC-32 (noise) } & \multicolumn{3}{|c|}{ TC-33 (blur) } \\
\hline & & $p=4$ & $p=8$ & $p=16$ & $p=4$ & $p=8$ & $p=16$ & $p=4$ & $p=8$ & $p=16$ & $p=4$ & $p=8$ & $p=16$ & $p=4$ & $p=8$ & $p=16$ \\
\hline \multirow[b]{2}{*}{2} & FGLAM & 89.71 & 90.88 & 91.18 & 91.08 & 92.15 & 93.21 & 91.76 & 93.75 & 93.68 & 92.57 & 89.26 & 85.51 & 97.72 & 98.46 & 99.34 \\
\hline & LBP & $\begin{array}{c}85.59 \\
S \_M\end{array}$ & $\begin{array}{c}M_{c} \\
87.94 \\
M S / C\end{array}$ & $\begin{array}{c}M_{c}-1 . l l \\
86.62 \\
S / M / C\end{array}$ & $\begin{array}{l}1 . I_{c} \\
86.44 \\
S M\end{array}$ & $\begin{array}{c}M_{c} \\
89.06 \\
M S / C\end{array}$ & $\begin{array}{c}M_{C} \\
90.71 \\
S / M / C\end{array}$ & $\begin{array}{l}M_{c} \\
67.50 \\
S . M\end{array}$ & $\begin{array}{l}921 \\
92.79 \\
S / M\end{array}$ & $\begin{array}{c}8 M_{C} \\
86.99\end{array}$ & 23.16 & $\begin{array}{l}M 7.87 \\
S / M\end{array}$ & 23.16 & $\frac{177}{49.93}$ & $\begin{array}{r}M 97 \\
99.49\end{array}$ & $\frac{111}{87.43}$ \\
\hline \multirow[b]{2}{*}{4} & FGLAM & $\begin{array}{c}89.56 \\
M_{c}\end{array}$ & $\begin{array}{c}90.29 \\
M_{c}\end{array}$ & $\begin{array}{c}91.32 \\
M_{l}\end{array}$ & $\begin{array}{c}90.75 \\
M_{c}\end{array}$ & $\begin{array}{c}92.10 \\
M_{c}\end{array}$ & $\begin{array}{c}93.06 \\
M_{c}\end{array}$ & $\begin{array}{c}91.99 \\
M_{c}\end{array}$ & $\begin{array}{c}93.75 \\
M_{c}\end{array}$ & $\begin{array}{c}\mathbf{9 3 . 7 5} \\
M_{c}\end{array}$ & $\begin{array}{c}91.03 \\
M_{l}\end{array}$ & $\begin{array}{c}89.34 \\
M_{l}\end{array}$ & $\begin{array}{c}86.10 \\
M_{l}\end{array}$ & $\begin{array}{c}97.87 \\
M_{l}\end{array}$ & $\begin{array}{c}99.49 \\
M_{l}\end{array}$ & $\overline{99.56}$ \\
\hline & LBP & $\begin{array}{c}83.24 \\
S \_M\end{array}$ & $\begin{array}{c}M_{c} \\
86.18 \\
M_{-} S / C\end{array}$ & $\begin{array}{c}81.62 \\
S\end{array}$ & $\begin{array}{l}82.67 \\
S \_M\end{array}$ & $\begin{array}{c}82.59 \\
M \_S / C\end{array}$ & $\begin{array}{c}84.71 \\
S / M / C\end{array}$ & $\begin{array}{l}71.25 \\
S \_M\end{array}$ & $\begin{array}{r}94.71 \\
S / M\end{array}$ & $\begin{array}{c}81.76 \\
S\end{array}$ & $\begin{array}{l}34.93 \\
S_{-} M\end{array}$ & $\begin{array}{l}62.06 \\
S / M\end{array}$ & $\begin{array}{c}52.94 \\
S\end{array}$ & $\begin{array}{l}77.50 \\
S_{-} M\end{array}$ & $\begin{array}{c}99.93 \\
S / M\end{array}$ & $\begin{array}{c}99.26 \\
S\end{array}$ \\
\hline
\end{tabular}

(c) FGLAMs $(p=16)$ vs. FLBPs and LFPs on gray-level and color datasets (Histogram: see section 5.3.3)

\begin{tabular}{|c|c|c|c|c|c|c|c|c|c|c|c|}
\hline$d$ & Descriptor & TC-13-1 & $\mathrm{TC}-20$ & $\mathrm{TC}-21$ & $\mathrm{TC}-22$ & $\mathrm{TC}-23$ & $\mathrm{TC}-13$ & $\mathrm{TC}-30$ & TC-31 & TC-32 & $\mathrm{TC}-33$ \\
\hline \multirow{4}{*}{2} & FGLAM $_{l}$ & 79.41 & 80.88 & 78.97 & 60.29 & 95.66 & 91.18 & 93.08 & 93.24 & 85.51 & 99.34 \\
\hline & FGLAM $_{c}$ & 80.15 & 82.51 & 80.07 & 56.69 & 92.43 & 91.18 & 93.21 & 93.68 & 81.47 & 97.06 \\
\hline & FLBP & 83.68 & 48.89 & 84.41 & 19.49 & 93.68 & 88.38 & 52.63 & 87.72 & 23.01 & 94.41 \\
\hline & LFP & 71.32 & 73.69 & 65.74 & 14.12 & 61.99 & 84.85 & 85.34 & 78.09 & 17.57 & 17.57 \\
\hline \multirow{4}{*}{4} & $\overline{\text { FGLAM }_{l}}$ & 77.79 & 79.65 & 77.35 & 62.35 & 95.66 & 91.32 & 92.93 & 93.46 & 86.10 & 99.56 \\
\hline & FGLAM $_{c}$ & 80.00 & 81.21 & 78.68 & 58.16 & 94.12 & 90.44 & 93.06 & 93.75 & 83.97 & 98.68 \\
\hline & FLBP & 79.41 & 45.62 & 83.16 & 50.66 & 100.00 & 83.97 & 49.47 & 85.96 & 55.37 & 100.00 \\
\hline & LFP & 72.79 & 72.68 & 65.22 & 29.19 & 85.88 & 82.06 & 83.21 & 75.96 & 36.84 & 36.84 \\
\hline $\mathrm{n} / \mathrm{a}$ & Histogram & 76.03 & 77.89 & 73.16 & 50.37 & 93.82 & 91.76 & 92.81 & 93.16 & 76.91 & 99.19 \\
\hline
\end{tabular}

- for $p=16, \mathrm{M}_{l}$ and $\mathrm{M}_{c}(l=256)$ vs. CLBP_S $S_{8, d}(l=256)$, CLBP $\_M_{8, d}(l=256)$, and CLBP_S $S_{P, R}^{r i u 2} / M_{P, R}^{r i u 2} / C(l=200)$.

Tables 5(a) and 5(b) show the classification results achieved on the ten gray-level and color Outex datasets by FGLAMs and LBP-based descriptors according to their sizes. For each given dataset, $p$ and $d$ values, the boldface result is the better one among the two families of descriptors, and the best descriptor in each family (either the cardinal or local-count FGLAM, and either of the three tested CLBP descriptors) is written below its result. Note that sub- and superscripts of CLBP descriptors are dropped in these tables to alleviate notations, and that their results slightly differ from those in [37] because we use the intersection instead of the $\chi^{2}$ distance to measure the similarity between two histograms.

FGLAMs perform strictly better than CLBP descriptors in 17 out of the 30 cases (56.6\%) for graylevel datasets and more remarkably in 27 out of the 30 cases (90.0\%) for color ones. FGLAMs noticeably outperform CLBPs on the dataset TC-22 degraded by noise, to which LBPs are poorly robust, and for 
very small descriptors $(p=4)$. FGLAMs also compare favorably with rotation-invariant CLBPs on the dataset TC-20, which shows that FGLAMs inherently handle rotation variations quite well.

\subsubsection{Comparison with other fuzzy texture descriptors}

We also compare the performances reached by FGLAMs with those provided by other fuzzy texture descriptors. The Fuzzy LBPs (FLBPs) proposed by Keramidas et al. [19] make each pixel's neighborhood partly contribute to several bins of the LBP histogram. The Local Fuzzy Patterns (LFPs) of Vieira et al. [38] represent each pixel's neighborhood as a fuzzy set defined by the membership degree of neighboring pixels to the central value. The sigmoid is used as membership function, which generalizes the step function of "crisp" LBPs. We test these two approaches on the Outex gray-level and color datasets with the optimal parameters given by their authors, but with the 1NN classification scheme as before. In order to obtain an equal size of 256 for FLBP and LFP histograms and for FGLAMs so that these three descriptors are fairly compared, we compute FGLAMs by setting $p=16$ and FLBPs with a 8-neighborhood instead of the full $3 \times 3$ neighborhood of [19]. Table 5(c) shows that LFPs are somewhat robust to rotation but are in general of minor interest. FLBP is a far more robust descriptor that notably shows superiority with regard to FGLAMs when the image resolution changes. However, FGLAMs perform better on color images. At last, we compare FGLAM performance with that of the Fuzzy Co-occurrence Matrices (FzCMs) developed by Munklang et al. [23]. This approach uses the fuzzy C-means algorithm to quantize the gray levels, then builds eight fuzzy GLCMs for each main direction of the image plane, and extracts the average and standard deviations of four Haralick's features from these matrices. For a fair comparison with results in [23], we implement the classification scheme based on the one-versus-all strategy of a multi-class Support Vector Machine (SVM) with $\sigma=0.25$ in the radial basis function kernel and $\epsilon=10^{-3}$ as termination criterion tolerance, and we similarly validate the classification thanks to a 10 -fold cross validation. We compare the texture classification results on the (challenging) UIUC dataset that contains 25 texture classes of 40 gray-level images [39]. Because the best classification accuracy of FzCMs (77.0\%) is reached with 64-dimensional feature vectors [23], we compute FGLAMs of size 64 by setting $p=8$. As $\mathrm{FGLAM}_{c}$ and $\mathrm{FGLM}_{l}$ provide accuracies of $82.7 \%$ and $79.9 \%$, FGLAMs outperform FzCMs with a SVM classifier.

\subsubsection{Descriptor complexity}

We simply assess the complexity of the proposed descriptors by their processing requirements. Table 6 displays the processing times required to compute a FGLAM from a gray-level image by ImageJ plugins [40] implemented on a $2.6 \mathrm{GHz}$ machine with $8 \mathrm{~GB}$ of RAM. It shows that the processing time nearly linearly depends on the number $p$ of fuzzy numbers. Furthermore, the processing times required by $\mathrm{FGLAM}_{l}$ and $\mathrm{FGLAM}_{c}$ are similar, as those needed by site-specific and spatially-invariant 
Table 6: Processing times required to FGLAMs from a gray-level image of size $128 \times 128$ pixels (averages over 100 images of TC-20 dataset).

\begin{tabular}{|c|c|c|c|c|c|}
\hline & $\begin{array}{l}\text { Membership } \\
\text { function }\end{array}$ & $\begin{array}{l}\text { Neighborhood } \\
\text { function }\end{array}$ & $\begin{array}{l}\text { Proce } \\
p=4\end{array}$ & $\begin{array}{l}\text { sing ti } \\
p=8\end{array}$ & $\begin{array}{l}\mathrm{e}(\mathrm{ms}) \\
p=16\end{array}$ \\
\hline \multirow{3}{*}{ 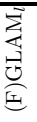 } & Crisp & $v$ & 23 & 41 & 76 \\
\hline & Gaussian & $\begin{array}{c}v \\
\dot{v}^{\mu}\end{array}$ & $\begin{array}{r}98 \\
100\end{array}$ & $\begin{array}{l}212 \\
218\end{array}$ & $\begin{array}{l}419 \\
430\end{array}$ \\
\hline & Triangular & $\begin{array}{c}v \\
\dot{v}^{\mu}\end{array}$ & $\begin{array}{l}2 \\
44\end{array}$ & $\begin{array}{l}78 \\
84\end{array}$ & $\begin{array}{l}150 \\
159\end{array}$ \\
\hline \multirow{3}{*}{ 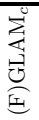 } & Crisp & $\bar{v}$ & 25 & 46 & 91 \\
\hline & Gaussian & $\begin{array}{c}v \\
\dot{v}^{\mu}\end{array}$ & $\begin{array}{l}114 \\
118\end{array}$ & $\begin{array}{l}247 \\
254\end{array}$ & $\begin{array}{l}492 \\
503\end{array}$ \\
\hline & Triangular & $\begin{array}{c}v \\
\dot{v}^{\mu}\end{array}$ & $\begin{array}{l}49 \\
52\end{array}$ & $\begin{array}{l}90 \\
95\end{array}$ & $\begin{array}{l}177 \\
185\end{array}$ \\
\hline
\end{tabular}

neighborhood functions. At last, fuzzy FGLAMs are more time-consuming than crisp ones notably when the Gaussian membership function is used. Besides, additional classification results for larger values of $p$ than in table 1 confirm that FGLAMs get similar to GLAMs as $p$ increases. Since our fuzzy descriptors bring almost no improvement with regard to crisp GLAMs when $p>16$ and require at least twice longer processing times, our approach is first and foremost interesting to compute very compact descriptors with a high discriminative power.

These relatively long processing times lead us to have a quick look back to very basic descriptors like histograms of luminance or RGB color components. Their classification performances are reported in the last row of table 5(c) and can be fairly compared with those of FGLAMs because all descriptors have the same size $\left(p^{2}=256\right.$ for gray-level histograms and $3 \times p^{2}=768$ for the three marginal RGB histograms). Histogram results are written in bold face when they outperform all other descriptors, which never occurs except for TC-13 color dataset as also reported in [32]. FGLAMs could hence be primarily interesting for color texture classification (with respect to a simple histogram) when the test subset is acquired under different conditions from the train subset. It is worth to investigate whether this also occurs on another database. Bianconi and Fernández point out that Outex is unfortunately the sole color texture database that comes with predefined test suites of training and test subsets [41]. We resort to the USPtex color texture dataset [42] (191 classes and 12 images by class acquired under the same conditions) with the same classification scheme (1-NN classifier and hold-out methodology) to further compare FGLAMs with histograms. The results (not shown here) are that the FGLAM $c$ descriptor computed with $\dot{v}^{\mu}$ always outperforms the marginal RGB histogram of the same size, by more than $10 \%$ when $p=16$. This confirms the benefit of our descriptors for texture classification, though it is less outstanding on color images than on gray-level and CFA ones.

\section{Conclusion and future work}

In this paper, we have extended the concept of aura set and the associated aura measure in several ways and we have experimentally shown the benefit of these contributions for texture classification on 
fifteen benchmark datasets of gray-level, color, and CFA images. We have first proposed a new aura measure based on the cardinal of the aura set that improves classification results on several datasets with regard to the original local-count aura measure. Second, we have adapted the aura concept to the fuzzy framework and demonstrated that fuzzy aura matrices generalize co-occurrence matrices. We have shown by extensive experiments that FGLAMs better characterize texture images than crisp matrices, than other powerful descriptors of similar sizes based on local binary patterns, and than other fuzzy descriptors in the literature. The proposed fuzzy measures of the aura set have been derived from their crisp counterparts but could be defined in other ways. Several definitions notably exist for the cardinality of fuzzy sets [43], and the fuzzy cardinal aura measure could take advantage of them.

Last but not least, we have detailed how the aura formalism provides an elegant way to deal with spatially-variant neighborhoods, as illustrated on CFA images. In this paper, we have used crisp neighborhoods (defined by a binary neighborhood function) that are simple but already provide promising results for texture classification tasks. In order to make neighborhood functions adapt to gray levels, we have retained a criterion based on the membership degrees of sites to gray-level sets, thus taking advantage of the fuzzy representation of neighborhood relationships. The definition of neighborhood functions should be investigated further than binary functions and than the empirical criterion of neighborhood adaptivity based on the membership degrees to fuzzy level sets. The design of more sophisticated functions and their adequacy to other purposes like texture segmentation is an open problem.

\section{Appendix}

A. Proof of $\tilde{m}_{l}\left(g, g^{\prime}\right) \geq \tilde{m}_{c}\left(g, g^{\prime}\right)$

Let $f_{\mathbf{r}}(\mathbf{s})=\min \left[\min \left(\mu_{S_{g}}(\mathbf{s}), v_{\mathbf{s}}(\mathbf{r})\right), \mu_{S_{g^{\prime}}}(\mathbf{r})\right]$ be a function of $\mathbf{s} \in \mathcal{S}$ for a given $\mathbf{r} \in \mathcal{S}$. Then, the aura measures of equations (15) and (18) is rewritten as $\tilde{m}_{l}\left(g, g^{\prime}\right)=\sum_{\mathbf{r} \in \mathcal{S}} \sum_{\mathbf{s} \in \mathcal{S}} f_{\mathbf{r}}(\mathbf{s})$ and $\tilde{m}_{c}\left(g, g^{\prime}\right)=$ $\sum_{\mathbf{r} \in \mathcal{S}} \sup _{\mathbf{s} \in \mathcal{S}} f_{r}(\mathbf{s}), 0 \leq g, g^{\prime} \leq q-1$. Because membership and neighborhood functions are positive, $f_{\mathbf{r}}(\mathbf{s}) \geq 0$ for all s. Therefore $\sum_{\mathbf{s} \in \mathcal{S}} f_{\mathbf{r}}(\mathbf{s}) \geq \sup _{\mathbf{s} \in \mathcal{S}} f_{\mathbf{r}}(\mathbf{s})$, hence $\tilde{m}_{l}\left(g, g^{\prime}\right) \geq \tilde{m}_{c}\left(g, g^{\prime}\right)$.

\section{B. FGLAMl as a FGLCM}

\section{B.1. Case of a single-site crisp neighborhood}

A fuzzy GLCM (FGLCM) [20, 22] is a $p \times p$ matrix $\tilde{C}=\left[\tilde{c}^{\mathrm{t}}\left(g, g^{\prime}\right)\right]$ whose element $\left(g, g^{\prime}\right)$ is the number of pairs of sites with gray levels "around the level $g$ " and "around the level $g$ " separated by a translation vector $\mathbf{t}$. In other words, it gives the number of occurrences for a pair of fuzzy numbers 
$\tilde{g}$ and $\tilde{g}^{\prime}$ as:

$$
\tilde{c}^{\mathbf{t}}\left(g, g^{\prime}\right)=\sum_{\mathbf{s} \in \mathcal{S}} \min \left\{\mu_{\tilde{g}}(I(\mathbf{s})), \mu_{\tilde{g}^{\prime}}(I(\mathbf{s}+\mathbf{t}))\right\} .
$$

In that case, the neighborhood can be considered as a crisp singleton and defined by the following binary neighborhood function:

$$
v_{\mathbf{s}}^{\mathbf{t}}(\mathbf{r})= \begin{cases}1 & \text { if } \mathbf{r}=\mathbf{s}+\mathbf{t} \\ 0 & \text { otherwise }\end{cases}
$$

Then, the local-count fuzzy aura measure is rewritten as:

$$
\begin{aligned}
\tilde{m}_{l}\left(g, g^{\prime}\right) & \stackrel{(15)}{=} \sum_{\mathbf{s} \in \mathcal{S}} \sum_{\mathbf{r} \in \mathcal{S}} \min \left\{\min \left[\mu_{S_{g}}(\mathbf{s}), v_{\mathbf{s}}^{\mathbf{t}}(\mathbf{r})\right], \mu_{S_{g^{\prime}}}(\mathbf{r})\right\} \\
& \stackrel{(28)}{=} \sum_{\mathbf{s} \in \mathcal{S}} \min \left\{\mu_{S_{g}}(\mathbf{s}), \mu_{S_{g^{\prime}}}(\mathbf{s}+\mathbf{t})\right\} \\
& \stackrel{(8)}{=} \sum_{\mathbf{s} \in \mathcal{S}} \min \left\{\mu_{\tilde{g}}(I(\mathbf{s})), \mu_{\tilde{g}^{\prime}}(I(\mathbf{s}+\mathbf{t}))\right\} \\
& \stackrel{(27)}{=} \tilde{c}^{\mathbf{t}}\left(g, g^{\prime}\right) .
\end{aligned}
$$

Since this holds for any gray levels $g$ and $g^{\prime}$, the local-count FGLAM is identical to the FGLCM $\left(\tilde{M}_{l} \equiv \tilde{C}\right)$ when the neighborhood function is binary and equals 1 for a single neighbor only.

\section{B.2. Case of a multi-site crisp neighborhood}

More generally, the neighborhood used to compute FGLAMs can be composed of several neighbors. As in the crisp case (see equation (4)), a FGLAM $l$ can then be written as a sum of $n$ "basic" local-count FGLAMs provided that the neighborhood function is a binary and only depends on $\mathbf{r}-\mathbf{s}$ :

$$
\tilde{m}_{l}\left(g, g^{\prime}\right)=\sum_{i=1}^{n} \tilde{c}^{\mathbf{t}_{i}}\left(g, g^{\prime}\right) \text {. }
$$

Justification. Let the neighborhood be $\mathcal{N}_{\mathbf{s}}=\bigcup_{i=1}^{n} \mathcal{N}_{\mathbf{s}}^{\mathbf{t}_{i}}$, where $\mathcal{N}_{\mathbf{s}}^{\mathbf{t}_{i}}$ is the basic neighborhood made of the single neighbor given by its translation $\mathbf{t}_{i}$ from $\mathbf{s}$ and defined by the neighborhood function $v_{\mathbf{s}}^{\mathbf{t}_{i}}(\mathbf{r})$. The neighborhood $\mathcal{N}_{\mathbf{s}}$ is then described by its neighborhood function $v_{\mathbf{s}}(\mathbf{r})=\sup _{i}\left(v_{\mathbf{s}}^{\mathbf{t}_{i}}(\mathbf{r})\right)=$ $\max \left(v_{\mathbf{s}}^{\mathbf{t}_{1}}(\mathbf{r}), \cdots, v_{\mathbf{s}}^{\mathbf{t}_{n}}(\mathbf{r})\right)$, and the fuzzy local-count aura measure of equation (15) is rewritten as:

$$
\tilde{m}_{l}\left(g, g^{\prime}\right)=\sum_{\mathbf{s} \in \mathcal{S}} \sum_{\mathbf{r} \in \mathcal{S}} \min \left\{\min \left[\mu_{S_{g}}(\mathbf{s}), \max \left(v_{\mathbf{s}}^{\mathbf{t}_{1}}(\mathbf{r}), \cdots, v_{\mathbf{s}}^{\mathbf{t}_{n}}(\mathbf{r})\right], \mu_{S_{g^{\prime}}}(\mathbf{r})\right\}\right.
$$

By using the properties of the min and $\max$ operators, the latter equation becomes: 


$$
\begin{aligned}
& \tilde{m}_{l}\left(g, g^{\prime}\right)=\sum_{\mathbf{s} \in \mathcal{S}} \sum_{\mathbf{r} \in \mathcal{S}} \min \left\{\max \left[\min \left(\mu_{S_{g}}(\mathbf{s}), v_{\mathbf{s}}^{\mathbf{t}_{1}}(\mathbf{r})\right), \cdots, \min \left(\mu_{S_{g}}(\mathbf{s}), v_{\mathbf{s}}^{\mathbf{t}_{n}}(\mathbf{r})\right)\right], \mu_{S_{g^{\prime}}}(\mathbf{r})\right\} \\
& =\sum_{\mathbf{s} \in \mathcal{S}} \sum_{\mathbf{r} \in \mathcal{S}} \max \left\{\min \left[\min \left(\mu_{S_{g}}(\mathbf{s}), v_{\mathbf{s}}^{\mathbf{t}_{1}}(\mathbf{r})\right), \mu_{S_{g^{\prime}}}(\mathbf{r})\right], \cdots, \min \left[\min \left(\mu_{S_{g}}(\mathbf{s}), v_{\mathbf{s}}^{\mathbf{t}_{n}}(\mathbf{r})\right), \mu_{S_{g^{\prime}}}(\mathbf{r})\right]\right\} \\
& \stackrel{(12)}{=} \sum_{\mathbf{s} \in \mathcal{S}} \sum_{\mathbf{r} \in \mathcal{S}} \max \left\{\min \left[\mu_{\mathcal{N}_{\mathbf{s}, S_{g}}^{\mathbf{t}_{1}}}, \mu_{S_{g^{\prime}}}(\mathbf{r})\right], \cdots, \min \left[\mu_{\mathcal{N}_{\mathbf{s}, S_{g}}^{t_{n}},}, \mu_{S_{g^{\prime}}}(\mathbf{r})\right]\right\} \\
& =\sum_{\mathbf{s} \in \mathcal{S}} \sum_{\mathbf{r} \in \mathcal{S}} \max \left\{\mu_{\mathcal{N}_{\mathbf{s}, S_{g}}^{\mathbf{t}_{1} \cap S_{g^{\prime}}}}, \cdots, \mu_{\mathcal{N}_{\mathbf{s}, S_{g}}^{\mathbf{t}_{n}} \cap S_{g^{\prime}}}\right\} \\
& =\sum_{\mathbf{s} \in \mathcal{S}} \sum_{\mathbf{r} \in \mathcal{S}} \mu_{\bigcup_{i=1}^{n}\left(\mathcal{N}_{\mathbf{s}, S_{g}}^{\mathbf{t}_{i}} \cap S_{g^{\prime}}\right)}(\mathbf{r}) \text {. }
\end{aligned}
$$

The cardinal of a union of fuzzy sets $A_{i}$ is expressed as:

$$
\begin{aligned}
\sum_{\mathbf{r} \in \mathcal{S}} \mu_{\left(\bigcup_{i=1}^{n} A_{i}\right)}(\mathbf{r}) & =\sum_{i=1}^{n} \sum_{\mathbf{r} \in \mathcal{S}} \mu_{A_{i}}(\mathbf{r})-\sum_{1 \leq i_{1}<i_{2} \leq n} \sum_{\mathbf{r} \in \mathcal{S}} \mu_{\left(A_{i_{1}} \cap A_{i_{2}}\right)}+\ldots \\
& +(-1)^{k+1} \sum_{1 \leq i_{1}<i_{2}<\ldots<i_{k} \leq n} \sum_{\mathbf{r} \in \mathcal{S}} \mu_{\left(A_{i_{1}} \cap A_{i_{2}} \cap \ldots \cap A_{i_{k}}\right)}(\mathbf{r})+\ldots \\
& +(-1)^{n+1} \sum_{\mathbf{r} \in \mathcal{S}} \mu_{\left(A_{1} \cap A_{2} \cap \ldots \cap A_{n}\right)}(\mathbf{r})
\end{aligned}
$$

Because the basic neighborhoods $\mathcal{N}_{\mathbf{s}}^{\mathbf{t}_{i}}$ do not overlap, the fuzzy sets $\mathcal{N}_{\mathbf{s}, S_{g}}^{\mathbf{t}_{i}} \cap S_{g^{\prime}}$ are disjoint for $i=$

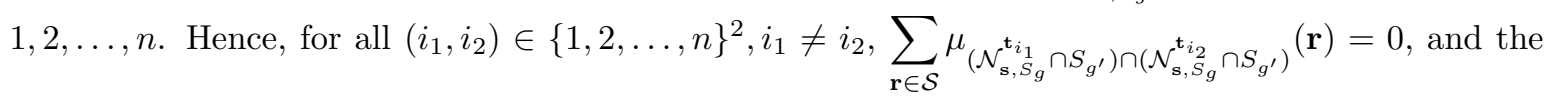
cardinal of the union of fuzzy set intersections in equation (30) becomes $\sum_{\mathbf{r} \in \mathcal{S}} \mu_{\bigcup_{i=1}^{n}\left(\mathcal{N}_{\mathbf{s}, S_{g}}^{\mathbf{t}_{i}} \cap S_{g^{\prime}}\right)}(\mathbf{r})=$ $\sum_{i=1}^{n} \sum_{\mathbf{r} \in \mathcal{S}} \mu_{\mathcal{N}_{\mathbf{s}, S g}^{\mathbf{t}_{i}} \cap S_{g^{\prime}}}(\mathbf{r})$. Therefore, equation (30) takes the form $\tilde{m}_{l}\left(g, g^{\prime}\right)=\sum_{\mathbf{s} \in \mathcal{S}} \sum_{i=1}^{n} \sum_{\mathbf{r} \in \mathcal{S}} \mu_{\mathcal{N}_{\mathbf{s}, S_{g}}^{\mathbf{t}_{i}} \cap S_{g^{\prime}}}(\mathbf{r})$ which, by swapping the first sums and by using equation (17), can be rewritten as:

$$
\tilde{m}_{l}\left(g, g^{\prime}\right)=\sum_{i=1}^{n} \sum_{\mathbf{s} \in \mathcal{S}} \sum_{\mathbf{r} \in \mathcal{S}} \min \left[\min \left(\mu_{S_{g}}(\mathbf{s}), v_{\mathbf{s}}^{\mathbf{t}_{i}}(\mathbf{r})\right), \mu_{S_{g^{\prime}}}(\mathbf{r})\right] .
$$

By setting $\tilde{c}^{\mathbf{t}_{i}}\left(g, g^{\prime}\right)=\sum_{\mathbf{s} \in \mathcal{S}} \sum_{\mathbf{r} \in \mathcal{S}} \min \left[\min \left(\mu_{S_{g}}(\mathbf{s}), v_{\mathbf{s}}^{\mathbf{t}_{i}}(\mathbf{r})\right), \mu_{S_{g^{\prime}}}(\mathbf{r})\right]$, we get equation (29).

We conclude that a FGLAM $l$ is identical to an "extended" FGLCM computed with a neighborhood given by a set of translation vectors. However, this is no longer true if the neighborhood function $v$ is not binary or/and actually depends on $\mathbf{r}$ and $\mathbf{s}$ because the neighborhood used to compute such an "extended" FGLCM is given by a binary neighborhood function defined identically at each site.

\section{References}

[1] M. Mirmehdi, X. Xie, J. Suri, Handbook of texture analysis, Imperial College Press, 2008. 
[2] X. Xie, M. Mirmehdi, A galaxy of texture features, in: M. Mirmehdi, X. Xie, J. Suri (Eds.), Handbook of texture analysis, Imperial College Press, 2008, Ch. 13, pp. 375-406.

[3] R. M. Haralick, K. Shanmugan, I. Dinstein, Textural features for image classification, IEEE Transactions on Systems, Man and Cybernetics 3 (6) (1973) 610-621.

[4] C. Palm, Color texture classification by integrative co-occurrence matrices, Pattern Recognition 37 (5) (2004) 965-976.

[5] I. M. Elfadel, R. W. Picard, Gibbs random fields, cooccurrences, and texture modeling, IEEE Transactions on Pattern Analysis and Machine Intelligence 16 (1) (1994) 24-37.

[6] X. Qin, Y.-H. Yang, Basic gray level aura matrices: theory and its application to texture synthesis, in: Procs. 10th International Conference on Computer Vision (ICCV'05), Vol. 1, Beijing, China, 2005, pp. 128-135.

[7] X. Qin, Y.-H. Yang, Aura 3D textures, IEEE Transactions on Visualization and Computer Graphics 13 (2) (2007) 379-389.

[8] X. Qin, Y.-H. Yang, Similarity measure and learning with gray level aura matrices (GLAM) for texture image retrieval, in: Procs. IEEE Computer Society Conf. on Computer Vision and Pattern Recognition (CVPR 2004), Vol. 1, IEEE Computer Society, Washington, DC, USA, 2004, pp. I-326-I-333.

[9] S. Wiesmüller, D. A. Chandy, Content based mammogram retrieval using gray level aura matrix, International Journal of Computer Communication and Information System 2 (1) (2010) 217-223.

[10] S. Liao, A. C. S. Chung, Texture classification by using advanced local binary patterns and spatial distribution of dominant patterns, in: Procs. IEEE International Conference on Acoustics, Speech and Signal Processing (ICASSP'07), Vol. 1, Honolulu, Hawai'i, U.S.A., 2007, pp. 1221-1224.

[11] Y. Wang, X. Gao, R. Fu, Y. Jian, Dayside corona aurora classification based on X-gray level aura matrices, in: Procs. 9th ACM International Conference on Image and Video Retrieval (CIVR 2010), Xi'an, China, 2010, pp. 282-287.

[12] M. A. Hannan, M. Arebey, R. A. Begum, H. Basri, Gray level aura matrix: An image processing approach for waste bin level detection, in: Procs. World Congress on Sustainable Technologies (WCST 2011), London, UK, 2011, pp. 77-82.

[13] M. Khalid, R. Yusof, A. S. M. Khairuddin, Improved tropical wood species recognition system based on multi-feature extractor and classifier, World Academy of Science, Engineering and Technology 59 (2011) 1702-1708. 
[14] M. A. Hannan, M. Arebey, R. A. Begum, H. Basri, An automated solid waste bin level detection system using a gray level aura matrix, Waste Management 32 (12) (2012) 2229-2238.

[15] R. Yusof, M. Khalid, A. S. M. Khairuddin, Application of kernel-genetic algorithm as nonlinear feature selection in tropical wood species recognition system, Computers and Electronics in Agriculture 93 (2013) 68-77.

[16] Z. Haliche, K. Hammouche, The gray level aura matrices for textured image segmentation, Analog Integrated Circuits and Signal Processing 69 (1) (2011) 29-38.

[17] P. Sobrevilla, E. Montseny, Fuzzy sets in computer vision: an overview, Mathware \& Soft Computing 10 (3) (2003) 71-83.

[18] A. Barcelo, E. Montseny, P. Sobrevilla, Fuzzy texture unit and fuzzy texture spectrum for texture characterization, Fuzzy Sets and Systems 158 (3) (2007) 239-252.

[19] E. Keramidas, D. Iakovidis, D. Maroulis, Fuzzy binary patterns for uncertainty-aware texture representation, Electronic Letters on Computer Vision and Image Analysis 10 (1) (2011) 63-78.

[20] C. Jawahar, A. Ray, Incorporation of gray-level imprecision in representation and processing of digital images, Pattern Recognition Letters 17 (5) (1996) 541-546.

[21] H. D. Cheng, C. H. Chen, H. H. Chiu, Image segmentation using fuzzy homogeneity criterion, Information Sciences 98 (1-4) (1997) 237-262.

[22] D. Sen, S. K. Pal, Image segmentation using global and local fuzzy statistics, in: Procs. IEEE India Council International Conference (INDICON 2006), New Delhi, India, 2006, pp. 1-6.

[23] Y. Munklang, S. Auephanwiriyakul, N. Theera-Umpon, A novel fuzzy co-occurrence matrix for texture feature extraction, in: B. Murgante, S. Misra, M. Carlini, C. M. Torre, H.-Q. Nguyen, D. Taniar, B. O. Apduhan, O. Gervasi (Eds.), Procs. 13th International Conference on Computational Science and Its Applications (ICCSA'2013), Lecture Notes in Computer Science, SpringerVerlag Berlin Heidelberg, Ho Chi Minh City, Vietnam, 2013, pp. 246-257.

[24] R. Verdú-Monedero, J. Angulo, J. Serra, Anisotropic morphological filters with spatially-variant structuring elements based on image-dependent gradient fields, IEEE Transactions on Image Processing 20 (1) (2011) 200-212.

[25] A. Landström, M. J. Thurley, Adaptive morphology using tensor-based elliptical structuring elements, Pattern Recognition Letters 34 (12) (2013) 1416-1422. 
[26] M. Syrjäsuo, E. F. Donovan, X. Qin, Y.-H. Yang, Automatic classification of auroral images in substorm studies, in: Procs. 8th International Conference on Substorms (ICS8), Banff Centre, Canada, 2006, pp. 309-313.

[27] D. Dubois, H. Prade, Fuzzy sets and systems: theory and applications, Vol. 144 of Mathematics in science and engineering, Academic Press, New York, 1980.

[28] M. Hanss, Applied fuzzy arithmetic: an introduction with engineering applications, SpringerVerlag Berlin Heidelberg, 2005.

[29] L. A. Zadeh, A theory of approximate reasoning machine intelligence, ed. by j. hayes, d. michie and l.i. mikulich, in: J. E. Hayes, D. Michie, L. I. Mikulich (Eds.), Machine Intelligence, Elsevier, Amsterdam, 1979, pp. 149-194.

[30] I. Bloch, H. Maître, Fuzzy mathematical morphologies: A comparative study, Pattern Recognition 28 (9) (1995) 1341-1387.

[31] Y. Rubner, J. Puzicha, C. Tomasi, J. M. Buhmann, Empirical evaluation of dissimilarity measures for color and texture, Computer Vision and Image Understanding 84 (1) (2001) 25-43.

[32] T. Mäenpää, M. Pietikäinen, Classification with color and texture: jointly or separately?, Pattern Recognition 37 (2004) 1629-1640.

[33] O. Losson, A. Porebski, N. Vandenbroucke, L. Macaire, Color texture analysis using CFA chromatic co-occurrence matrices, Computer Vision and Image Understanding 117 (7) (2013) 747-763.

[34] M. Pietikäinen, A. Hadid, G. Zhao, T. Ahonen, Computer vision using local binary patterns, Vol. 40 of Computational Imaging and Vision, Springer-Verlag London, 2011.

[35] T. Ojala, T. Mäenpää, M. Pietikäinen, J. Viertola, J. Kyllönen, S. Huovinen, Outex new framework for empirical evaluation of texture analysis algorithms, in: Procs. 16th International Conference on Pattern Recognition, Vol. 1, 2002, pp. 701-706. Available from: http://www. outex. oulu.fi/temp.

[36] T. Ojala, M. Pietikäinen, T. Mäenpää, Multiresolution gray-scale and rotation invariant texture classification with local binary patterns, IEEE Transactions on Pattern Analysis and Machine Intelligence 24 (7) (2002) 971-987.

[37] Z. Guo, L. Zhang, D. Zhang, A completed modeling of local binary pattern operator for texture classification, IEEE Transactions on Image Processing 19 (6) (2010) 1657-1663. 
[38] R. T. Vieira, C. E. de Oliveira Chierici, C. T. Ferraz, A. Gonzaga, Local fuzzy pattern: A new way for micro-pattern analysis, in: H. Yin, J. A. F. Costa, G. Barreto (Eds.), Intelligent Data Engineering and Automated Learning - IDEAL 2012, Vol. 7435 of Lecture Notes in Computer Science, Springer Berlin Heidelberg, 2012, pp. 602-611.

[39] S. Lazebnik, C. Schmid, J. Ponce, A sparse texture representation using local affine regions, IEEE Transactions on Pattern Analysis and Machine Intelligence 27 (8) (2005) 1265-1278. Available from: http://www-cvr.ai.uiuc.edu/ponce_grp/data/\#texture.

[40] W. S. Rasband, ImageJ, U. S. National Institutes of Health, Bethesda, MD, USA (1997-2015). Available from: http://imagej.nih.gov/ij/.

[41] F. Bianconi, A. Fernández, An appendix to "Texture databases - A comprehensive survey", Pattern Recognition Letters 45 (2014) 33-38.

[42] A. R. Backes, D. Casanova, O. M. Bruno, Color texture analysis based on fractal descriptors, Pattern Recognition 45 (5) (2012) 1984-1992. Available from: http://fractal.ifsc.usp.br/ dataset/USPtex.php.

[43] M. Dhar, On cardinality of fuzzy sets, International Journal of Intelligent Systems and Applications 5 (6) (2013) 47-52. 